\title{
Nlrp3 Inflammasome Genetic Variants Are Associated With Risk And/Or Protection To Hospitalization Among Covid-19 Patients From Rio De Janeiro, Brazil
}

Nathalia Beatriz Ramos de Sá ( $\nabla$ nathalia.beatriz2008@gmail.com )

Laboratory of AIDS \& Molecular Immunology, Oswaldo Cruz Institute, FIOCRUZ, Rio de Janeiro

Milena Neira-Goulart

Laboratory of AIDS \& Molecular Immunology, Oswaldo Cruz Institute, FIOCRUZ, Rio de Janeiro

Marcelo Ribeiro-Alves

Laboratory of Clinical Research on STD/AIDS, National Institute of Infectology Evandro Chagas, FIOCRUZ, Rio de Janeiro

Kim Mattos Geraldo

Laboratory of Clinical Research on STD/AIDS, National Institute of Infectology Evandro Chagas,

FIOCRUZ, Rio de Janeiro

\section{Maria Pia Diniz Ribeiro}

Laboratory of Clinical Research on STD/AIDS, National Institute of Infectology Evandro Chagas, FIOCRUZ, Rio de Janeiro

\section{Sandra Wagner Cardoso}

Laboratory of Clinical Research on STD/AIDS, National Institute of Infectology Evandro Chagas, FIOCRUZ, Rio de Janeiro

\section{Beatriz Grinsztejn}

Laboratory of Clinical Research on STD/AIDS, National Institute of Infectology Evandro Chagas, FIOCRUZ, Rio de Janeiro

\section{Valdiléa G. Veloso}

Laboratory of Clinical Research on STD/AIDS, National Institute of Infectology Evandro Chagas, FIOCRUZ, Rio de Janeiro

\section{Artur Capão}

Laboratory of Respiratory Virus and Measles, Oswaldo Cruz Institute, FIOCRUZ, Rio de Janeiro

\section{Marilda Mendonça Siqueira}

Laboratory of Respiratory Virus and Measles, Oswaldo Cruz Institute, FIOCRUZ, Rio de Janeiro

\section{Ohanna Cavalcanti de Lima Bezerra}

Laboratory of Respiratory Virus and Measles, Oswaldo Cruz Institute, FIOCRUZ, Rio de Janeiro

\section{Cristiana Couto Garcia}

Laboratory of Respiratory Virus and Measles, Oswaldo Cruz Institute, FIOCRUZ, Rio de Janeiro 


\section{Larissa Rodrigues Gomes}

Center of Technological Development in Health (CDTS)/National Institute of Science and Technological for Innovation on Neglected population diseases (INCT-IDPN), FIOCRUZ, Rio de Janeiro

\section{Andressa da Silva Cazote}

Laboratory of AIDS \& Molecular Immunology, Oswaldo Cruz Institute, FIOCRUZ, Rio de Janeiro

Dalziza Victalina De Almeida

Laboratory of AIDS \& Molecular Immunology, Oswaldo Cruz Institute, FIOCRUZ, Rio de Janeiro

Carmem Beatriz Wagner Giacoia-Gripp

Laboratory of AIDS \& Molecular Immunology, Oswaldo Cruz Institute, FIOCRUZ, Rio de Janeiro

\section{Fernanda Heloise Côrtes}

Laboratory of AIDS \& Molecular Immunology, Oswaldo Cruz Institute, FIOCRUZ, Rio de Janeiro

\section{Mariza Gonçalves Morgado}

Laboratory of AIDS \& Molecular Immunology, Oswaldo Cruz Institute, FIOCRUZ, Rio de Janeiro

\section{Research Article}

Keywords: COVID-19, Inflammasome single nucleotide polymorphisms (SNPs), risk factors

Posted Date: November 22nd, 2021

DOI: https://doi.org/10.21203/rs.3.rs-1050492/v1

License: (c) (i) This work is licensed under a Creative Commons Attribution 4.0 International License. Read Full License 


\section{Abstract \\ Background}

COVID-19 has a broad spectrum of clinical manifestations, from asymptomatic to mild or moderate symptoms, reaching the most severe forms and death. The mechanisms underlying the SARS-CoV-2 infection and its clinical evolution are still unclear. Once SARS-CoV-2 infects individuals, host factors are activated by the presence of the virus inside the cells, such as the inflammasome system. The search of risk factors for COVID-19 is of relevance for clinical management. In this study, we investigated the impact of 11 single-base polymorphisms (SNPs) in the NLRP3, CARD8, AIM2, CASP-1, IFI16, and IL-1 $\beta$ inflammasome genes in SARS-CoV-2 infected individuals with distinct disease outcomes.

\section{Methods}

Patients were divided into two groups: (1) inpatients, with severe/critical disease (Hospitalized group, $\mathrm{n}=451$ ), and (2) convalescent volunteers with prior SARS-CoV-2 infection and a history of asymptomatic to mild symptoms (Mild group, $n=43$ ). Patients hospitalized were followed up at a Hospital Center for COVID-19 Pandemic - National Institute of Infectology (INI)/FIOCRUZ, Rio de Janeiro, Brazil, from June 2020 to March 2021. The Mild group was recruited at Oswaldo Cruz Institute (IOC)/FIOCRUZ, Rio de Janeiro, Brazil, in 2020. Genotyping of the SNPs was determined by Real-Time PCR. Protection and risk estimations were performed by unconditional logistic regression models.

\section{Results}

Among the genotyped SNPs, significant differences in the NLRP3 rs1539019 and rs10754558 frequencies were observed between the groups. The $\mathrm{C} / \mathrm{C}$ genotype $\left(\mathrm{OR}_{\mathrm{adj}}=6.31 ; P_{\mathrm{adj}}=0.026\right)$ or allele $\mathrm{C}$ $\left(\mathrm{OR}_{\mathrm{adj}}=1.05 ; P_{\mathrm{adj}}=0.002\right)$ in rs1539019 polymorphism were associated with the risk for hospitalization, while the $\mathrm{C} / \mathrm{G}$ genotype $\left(\mathrm{OR}_{\mathrm{adj}}=0.16 ; P_{\mathrm{adj}}=0.016\right)$ or carrier-G $\left(\mathrm{OR}_{\mathrm{adj}}=0.2 ; P_{\mathrm{adj}}=0.028\right)$ in $\mathrm{rs} 10754558$ polymorphism were associated with protection for hospitalization. Regarding the NLRP3 genetic variants, the A-C-G-C-G haplotype $\left(\mathrm{OR}_{\mathrm{adj}}=0.14 ; P_{\mathrm{adj}}=0.030\right)$ was associated with protection for hospitalization. No significant association was observed for the other polymorphisms.

\section{Conclusions}

As of our knowledge, this is the first study demonstrating the association of inflammasome NLRP3 variants with risk and/or protection for hospitalization in COVID-19. Studies linking the NLRP3 inflammasome and SARS-CoV-2 infection are still scarce due to the recent emergence of this pathogen. Our results contribute to the discussion of the impact of inflammasomes in the clinical evolution of COVID-19. 


\section{Background}

Since the end of 2019/early 2020, a new disease emerged, described initially as an outbreak of viral pneumonia in individuals living in Wuhan, China (1). Researchers identified a new coronavirus as the pathogen causing the outbreak and named it as SARS-CoV-2 (2). The World Health Organization (WHO) called the associated coronavirus disease COVID-19 (3). Due to this fast dispersion around the world, on March 11, 2020, WHO raised COVID-19 to the category of a pandemic that is still ongoing (4). Globally, mortality and incidence of SARS-CoV-2 have increased rapidly. The Americas are the continents most affected by the COVID-19 pandemic now, with the United States of America (USA) and Brazil being the leaders in the numbers of cases to date (5). SARS-CoV-2 has already infected more than 230 million individuals worldwide, and 4.7 million patients have died, according to WHO (6). In Brazil, the first case was notified on February 26th, 2020, and the first community transmission was identified on March 13th, 2020. The country has accumulated more than 20 million reported cases, with more than 586,000 deaths as of September 13th, $2021(6,7)$.

COVID-19 has a broad spectrum of clinical manifestations, from asymptomatic to mild/moderate symptoms, to most critical forms, as a severe acute respiratory syndrome (SARS), thromboembolism, sepsis, multiple organ failure, and death (6). Though the risk of death due to the COVID-19 varies among the countries, age, comorbidities, and host genetic factors, the mechanisms underlying SARS-CoV-2 infection and its clinical evolution are still unclear $(8,9)$.

Once SARS-CoV-2 infects individuals, host factors are activated by the presence of the virus inside the cells. Pattern-recognition receptors (PRR), recognize conserved virus fragments, known as pathogenassociated molecular patterns (PAMP), and trigger the activation of several cellular components $(10,11)$. Among the large family of PRRs are NOD-like receptors (NLR), retinoic acid-inducible gene-I (RIG-I)-like receptors (RLRs) and, Toll-like receptors (TLRs) (12). Some studies have already shown that the RLRs family is an important PRR in the detection of coronaviruses $(13,14)$. Besides that, the NLR receptors stand out due to their wide recognition of intrinsic or extrinsic stimuli, operating principally as cytoplasmatic sensors (15). These receivers when activated lead to the NF-kB activation pathway, which culminates in the transcription of several molecules, such as gasdermin-D (GSDM-D), pro-IL-1b, and proIL-18, among others $(16,17)$. These released molecules cause a wave of local inflammation, involving increased secretion of proinflammatory cytokines and chemokines (e.g., IL-6, IFN-ץ, CCL2, and CXCL10) $(18,19)$. These and other cytokines have already been observed to be increased in SARS-Cov-2 infection, especially in more severe cases $(18,20)$.

The primary function of the NLR is to form a multiprotein complex, known as inflammasome. The inflammasomes are cytosolic multiprotein oligomers of the innate immune system responsible for the activation of inflammatory responses, which interact with several adapter proteins leading to the activation of caspase-1 and inducing the release of the proinflammatory cytokines IL-1ß and IL-18 (21). Different PRR (e.g., NLRP1 and NLRP3) can activate inflammasome assembly in response to specific stimuli, leading to inflammation and thrilling the innate immune response. Inflammasome activation is 
strictly regulated by endogenous host proteins (e.g., CARD8 and HSP90) and by a variety of transcriptional and post-transcriptional mechanisms (22). Inflammasomes belonging to the NLR are composed of at least three components: a protein sensor (e.g., NLRP1 and NLRP3), an inflammatory caspase (e.g., caspase-1 and caspase-11), and, finally, an adapter molecule containing a CARD domain (e.g., ASC) (23). It has been shown that proteins E, ORF3a e ORF8b from SARS-CoV activate NLRP3 inflammasome (15). Mutations in the inflammasome genes may lead to inflammatory disorders. For example, the constitutively IL-1 $\beta$ high levels contribute to chronic inflammation (24), including viral infections (25). SNPs in the NLRP3 gene were associated with a group of inflammatory disorders of genetic origin with the exaggerated secretion of IL-1 $\beta$ (26).

Studies have already observed the relationship between inflammasome activation and COVID-19 (12, 2730). Inflammasome activation is one of the main theories for the explanation of the cytokine storm during COVID-19 causing severe disease $(12,30)$. NLRP3 activation in COVID-19 patients has already been described in tissues of COVID-19 patients. Additionally, higher levels of IL-18 and Casp1p20 (Inflammasome products) in COVID-19 patients were associated with severe disease (30). However, exploring the role of the inflammasomes in the SARS-CoV-2 infection is still needed due to the recent onset of this pathogen. Genetic factors contributing to the outcome of SARS-CoV-2 infection are still little known; recently, variants in the specific sites of the ACE2 and TMPRSS2 genes, as well as the ABO locus and fewer others gene targets were considered as genetic risk factors for COVID-19 outcomes (31-33). No variants in inflammasome genes have ever been associated with risk or protection for COVID-19. The search of risk and/or protection factors for COVID-19 is of relevance for clinical management. Thus, in the present study, we investigated the impact of 11 single-base polymorphisms (SNPs) of the NLRP3, CARD8, AIM2, CASP-1, IFI16, and IL-1 $\beta$ inflammasome genes in SARS-CoV-2 infected individuals.

\section{Methods}

This is a case-control study nested in the RECOVER-SUS (Collaborative Network to Generate COVID-19 scientific evidence for the Brazilian Unified Health System), a cohort of individuals hospitalized with severe/critical COVID-19 at "Instituto Nacional de Infectologia Evandro Chagas" of the Oswaldo Cruz Foundation (INI-FIOCRUZ). In this study, the "Hospitalized Group" was formed by 451 individuals, a subset of the patients enrolled in the RECOVER-SUS cohort from June 2020 to March 2021. The "Mild Group" was formed with 43 individuals with RT-PCR confirmed SARS-CoV-2 infection with asymptomatic or mild disease severity, selected from the individuals tested for COVID-19 in 2020 by the Laboratory of Respiratory Virus and Measles - IOC/FIOCRUZ, Rio de Janeiro, Brazil, in 2020. The local Ethics Committee approved the studies. All participants signed an informed consent form.

Demographic and clinical data and blood samples were collected at the study entry visit. Skin color was self-declared following the classification system employed by the Brazilian Institute of Geography and Statistics (IBGE) (34). IBGE is the entity linked to the Brazilian Federal Government, responsible for collecting Brazilian statistical, geographic, cartographic, geodetic, and environmental information. 


\section{Genomic DNA extraction}

DNA was extracted from whole blood using the QIAamp DNA Blood Mini Kit (Qiagen, Hilden, NordrheinWestfalen, Germany), following the manufacturer's instructions. The DNA concentration was determined using a Thermo Scientific NanoDrop 2000 spectrophotometer (Thermo Fisher Scientific, Waltham, Massachusetts, USA). The filtrates containing the isolated DNA were stored at $-20^{\circ} \mathrm{C}$ until the genomics analyses.

\section{Single nucleotide polymorphism selection and genotyping}

We selected 11 SNPs in six inflammasome genes, considering the relevance of each gene in the inflammasome pathway: CARD8 (rs2043211, rs6509365), AIM2 (rs2276405), IFI16 (rs1101996), CASP-1 (rs572687), IL-1ß (rs1143634), and NLRP3 (rs10754558, rs1539019, rs4612666, rs3806268, and rs35829419). These were selected based primarily on a literature review about inflammasome SNPs in viral diseases. SNP genotyping was performed using commercially available TaqMan assays (Applied Biosystems/AB and Life Technologies) at the ABI7500 Real-Time platform (Applied Biosystems/AB and Life Technologies). Allelic discrimination was carried out employing the Thermo Fisher Connect Software. The SNP characteristics are listed in Table S1.

\section{Statistical analyses}

Mann-Whitney U tests were used to compare baseline demographic and clinical, continuous numerical variables, and Fisher's exact tests were used for categorical variables. In the SNP analyses, the frequencies of genotypes were determined by direct count, and deviations from HWE were assessed by $\chi 2$ tests. Pairwise LD patterns were determined for each gene using $r^{2}$ statistics (cut-off of $r^{2} \geq 0.8$ ). The homozygous genotypes of the allele with the major frequency in our sample were compared with the genotypes including the minor allele frequency allele (carriers) to better observe the differences caused by the variation. The protection/risk estimate was presented as adjusted odds ratios (aOR) with $95 \% \mathrm{Cl}$ for each SNP and estimated through unconditional logistic regression models. We included any clinical phenotypic marker associated with COVID-19 as confounders in modeling all other genotypic analyses to eliminate any possible bias. Haplotype frequencies were estimated by maximum likelihood and phase uncertainty was included in statistical models applied for association analyses. The most frequent haplotype of the NLRP3 and the CARD8 genes were considered references for the haplotype analyses. Multiple comparisons were corrected by the estimations of false-discovery rates (FDR). All statistical analyses were performed using $\mathrm{R}$ version 4.1.1 (R Core Team, 2021).

\section{Results}

\section{Sociodemographic and clinical characteristics of Hospitalized patients and Mild symptom controls}


The sociodemographic and clinical characteristics of the 494 individuals included in the present study are depicted in Table 1. The mean age was 58.05 years (IQR=21.79), with a mean of 41.06 years $(\mathrm{IQR}=18.46)$ in the Mild group and 60.25 years $(\mathrm{IQR}=21.84)$ in the Hospitalized group. Overall, 256 $(51.8 \%)$ individuals were male, 16 (37.2\%) from the Mild group, and 240 (53.2\%) from the Hospitalized group. Regarding schooling, 145 (29.4\%) of the individuals have a high school degree, with $5(11.6 \%)$ in the Mild group and 140 (38.2\%) in the Hospitalized group. The schooling, gender, skin color, age, comorbidities, and symptoms differed significantly between the Mild and Hospitalized groups. After correction by age, gender, skin color, schooling, and diabetes mellitus and coronary artery disease comorbidities, wherever applicable, only brown color, high school level, and nausea were significantly different between groups. 
Table 1

Sociodemographic and clinical features associated with either presentation of mild symptoms (mild group) or moderate/severe symptoms (hospitalized group) ( $\mathrm{N}=494)$.

\begin{tabular}{|c|c|c|c|c|c|c|c|}
\hline \multirow[t]{3}{*}{ Features } & & \multicolumn{2}{|l|}{ Outcomes } & \multirow{3}{*}{$\begin{array}{l}\text { OR } \\
\text { (Cl95\%) }\end{array}$} & \multirow{3}{*}{$\begin{array}{l}P \\
\text { value }^{\mathrm{b}}\end{array}$} & \multirow{3}{*}{$\begin{array}{l}\mathrm{aOR}^{\mathrm{a}} \\
\text { (Cl95\%) }\end{array}$} & \multirow{3}{*}{$\begin{array}{l}\text { Adjusted } \\
\text { Pvalue }^{\mathrm{b}}\end{array}$} \\
\hline & & \multirow{2}{*}{$\begin{array}{l}\text { Mild group } \\
\mathrm{N}=43\end{array}$} & \multirow{2}{*}{$\begin{array}{l}\text { Hospitalized } \\
\text { group } \\
\mathrm{N}=451\end{array}$} & & & & \\
\hline & & & & & & & \\
\hline \multirow[t]{2}{*}{ Gender; n (\%) } & female & $\begin{array}{l}27 \\
(62.79 \%)\end{array}$ & $\begin{array}{l}211 \\
(46.78 \%)\end{array}$ & Reference & & & \\
\hline & male & $\begin{array}{l}16 \\
(37.21 \%)\end{array}$ & $\begin{array}{l}240 \\
(53.22 \%)\end{array}$ & $\begin{array}{l}1.92 \\
(1.01- \\
3.66)\end{array}$ & 0.048 & $\begin{array}{l}3.26 \\
(0.97- \\
10.93)\end{array}$ & 1 \\
\hline \multirow[t]{3}{*}{$\begin{array}{l}\text { Skin Color; n } \\
(\%)\end{array}$} & white & $\begin{array}{l}31 \\
(72.09 \%)\end{array}$ & 75 (16.63\%) & Reference & & & \\
\hline & brown & 9 (20.93\%) & $\begin{array}{l}274 \\
(60.75 \%)\end{array}$ & $\begin{array}{l}12.58 \\
(5.74- \\
27.58)\end{array}$ & $<0.001$ & $\begin{array}{l}11.17 \\
(2.93- \\
42.61)\end{array}$ & 0.033 \\
\hline & other & $3(6.98 \%)$ & $\begin{array}{l}102 \\
(22.62 \%)\end{array}$ & $\begin{array}{l}14.05 \\
(4.14- \\
47.7)\end{array}$ & $<0.001$ & $\begin{array}{l}17.83 \\
(1.76- \\
180.3)\end{array}$ & 0.587 \\
\hline \multirow[t]{4}{*}{ Age; n (\%) } & $(18-40]$ & $\begin{array}{l}18 \\
(48.65 \%)\end{array}$ & 45 (10.92\%) & Reference & & & \\
\hline & $(40-60]$ & $\begin{array}{l}15 \\
(40.54 \%)\end{array}$ & $\begin{array}{l}159 \\
(38.59 \%)\end{array}$ & $\begin{array}{l}4.24 \\
(1.98- \\
9.07)\end{array}$ & $<0.001$ & $\begin{array}{l}1.68 \\
(0.18- \\
15.87)\end{array}$ & 1 \\
\hline & $(60-80]$ & $4(10.81 \%)$ & $\begin{array}{l}176 \\
(42.72 \%)\end{array}$ & $\begin{array}{l}17.6 \\
(5.68- \\
54.58)\end{array}$ & $<0.001$ & $\begin{array}{l}3.81 \\
(0.05- \\
278.1)\end{array}$ & 1 \\
\hline & $(80$ - 90.6] & $0(0 \%)$ & $32(7.77 \%)$ & NC & & & \\
\hline \multirow[t]{3}{*}{$\begin{array}{l}\text { Schooling; n } \\
(\%)\end{array}$} & $\begin{array}{l}\text { University } \\
\text { education }\end{array}$ & $\begin{array}{l}37 \\
(86.05 \%)\end{array}$ & $51(13.93 \%)$ & Reference & & & \\
\hline & $\begin{array}{l}\text { High } \\
\text { school }\end{array}$ & $5(11.63 \%)$ & $\begin{array}{l}140 \\
(38.25 \%)\end{array}$ & $\begin{array}{l}20.31 \\
(7.57- \\
54.52)\end{array}$ & $<0.001$ & $\begin{array}{l}13.22 \\
(3.25- \\
53.71)\end{array}$ & 0.025 \\
\hline & $\begin{array}{l}\text { Low } \\
\text { Education }\end{array}$ & 1 (2.33\%) & $\begin{array}{l}175 \\
(47.81 \%)\end{array}$ & $\begin{array}{l}126.96 \\
(17.01- \\
947.41)\end{array}$ & $<0.001$ & $\begin{array}{l}58.44 \\
(4.32- \\
789.95)\end{array}$ & 0.088 \\
\hline
\end{tabular}

aOdds ratios were adjusted by skin color, schooling, gender, age, and associated comorbidities such as diabetes mellitus and coronary artery disease. ${ }^{b} P$-values were calculated using the unconditional logistic regression model. Associations were considered significant with a value of * $P<0.05$. N: number of individuals in each group; aOR: adjusted odds ratio; $95 \% \mathrm{Cl}: 95 \%$ confidence interval; NC: not calculated. 


\begin{tabular}{|c|c|c|c|c|c|c|c|}
\hline \multirow[t]{3}{*}{ Features } & & \multicolumn{2}{|l|}{ Outcomes } & \multirow{3}{*}{$\begin{array}{l}\text { OR } \\
\text { (CI95\%) }\end{array}$} & \multirow{3}{*}{$\begin{array}{l}P \\
\text { value }^{\mathrm{b}}\end{array}$} & \multirow{3}{*}{$\begin{array}{l}\text { aOR } \\
\text { (Cl95\%) }\end{array}$} & \multirow{3}{*}{$\begin{array}{l}\text { Adjusted } \\
P_{\text {-value }}\end{array}$} \\
\hline & & \multirow{2}{*}{$\begin{array}{l}\text { Mild group } \\
\mathrm{N}=43\end{array}$} & $\begin{array}{l}\text { Hospitalized } \\
\text { group }\end{array}$ & & & & \\
\hline & & & $\mathrm{N}=451$ & & & & \\
\hline \multirow{2}{*}{$\begin{array}{l}\text { Diabetes } \\
\text { Mellitus; } \mathrm{n} \\
(\%)\end{array}$} & No & $\begin{array}{l}41 \\
(9535 \%)\end{array}$ & 313 (69.4\%) & Reference & & & \\
\hline & Yes & $2(4.65 \%)$ & 138 (30.6\%) & $\begin{array}{l}9.04 \\
(2.16- \\
37.87)\end{array}$ & 0.002 & $\begin{array}{l}5.7 \\
(0.6- \\
54.19)\end{array}$ & 1 \\
\hline \multirow{2}{*}{$\begin{array}{l}\text { Coronary } \\
\text { Artery } \\
\text { Disease; n } \\
(\%)\end{array}$} & No & 35 (81.4\%) & $\begin{array}{l}440 \\
(97.56 \%)\end{array}$ & Reference & & & \\
\hline & Yes & $8(18.6 \%)$ & $11(2.44 \%)$ & $\begin{array}{l}0.11 \\
(0.04- \\
0.29)\end{array}$ & $<0.001$ & $\begin{array}{l}0.01(0- \\
0.22)\end{array}$ & 0.197 \\
\hline \multirow{2}{*}{$\begin{array}{l}\text { Current } \\
\text { Smoking; n } \\
(\%)\end{array}$} & No & $\begin{array}{l}40 \\
(93.02 \%)\end{array}$ & $\begin{array}{l}427 \\
(94.68 \%)\end{array}$ & Reference & & & \\
\hline & Yes & $3(6.98 \%)$ & $24(5.32 \%)$ & $\begin{array}{l}0.75 \\
(0.22- \\
2.6)\end{array}$ & 0.649 & $\begin{array}{l}0.99 \\
(0.07- \\
14.8)\end{array}$ & 1 \\
\hline \multirow[t]{2}{*}{ Fever; n (\%) } & No & $\begin{array}{l}25 \\
(58.14 \%)\end{array}$ & $\begin{array}{l}208 \\
(46.12 \%)\end{array}$ & Reference & & & \\
\hline & Yes & $\begin{array}{l}18 \\
(41.86 \%)\end{array}$ & $\begin{array}{l}243 \\
(53.88 \%)\end{array}$ & $\begin{array}{l}1.62 \\
(0.86- \\
3.06)\end{array}$ & 0.134 & $\begin{array}{l}1.18 \\
(0.36- \\
3.84)\end{array}$ & 1 \\
\hline \multirow[t]{2}{*}{ Cough; n (\%) } & No & $\begin{array}{l}22 \\
(51.16 \%)\end{array}$ & $\begin{array}{l}176 \\
(39.02 \%)\end{array}$ & Reference & & & \\
\hline & Yes & $\begin{array}{l}21 \\
(48.84 \%)\end{array}$ & $\begin{array}{l}275 \\
(60.98 \%)\end{array}$ & $\begin{array}{l}1.64 \\
(0.87- \\
3.06)\end{array}$ & 0.123 & $\begin{array}{l}3.36 \\
(1.04- \\
10.85)\end{array}$ & 1 \\
\hline $\begin{array}{l}\text { Chest Pain; n } \\
(\%)\end{array}$ & No & $\begin{array}{l}34 \\
(79.07 \%)\end{array}$ & $\begin{array}{l}411 \\
(91.13 \%)\end{array}$ & Reference & & & \\
\hline
\end{tabular}

${ }^{a}$ Odds ratios were adjusted by skin color, schooling, gender, age, and associated comorbidities such as diabetes mellitus and coronary artery disease. ${ }^{\mathrm{b}} P$-values were calculated using the unconditional logistic regression model. Associations were considered significant with a value of * $P<0.05$. N: number of individuals in each group; aOR: adjusted odds ratio; $95 \% \mathrm{Cl}$ : $95 \%$ confidence interval; NC: not calculated. 


\begin{tabular}{|c|c|c|c|c|c|c|c|}
\hline \multicolumn{2}{|l|}{ Features } & \multicolumn{2}{|l|}{ Outcomes } & \multirow{3}{*}{$\begin{array}{l}\text { OR } \\
\text { (CI95\%) }\end{array}$} & \multirow{3}{*}{$\begin{array}{l}P \\
\text { value }^{\mathrm{b}}\end{array}$} & \multirow{3}{*}{$\begin{array}{l}\mathrm{aOR}^{\mathrm{a}} \\
\text { (Cl95\%) }\end{array}$} & \multirow{3}{*}{$\begin{array}{l}\text { Adjusted } \\
P_{\text {-value }}\end{array}$} \\
\hline & & \multirow{2}{*}{$\begin{array}{l}\text { Mild group } \\
\mathrm{N}=43\end{array}$} & $\begin{array}{l}\text { Hospitalized } \\
\text { group }\end{array}$ & & & & \\
\hline & & & $\mathrm{N}=451$ & & & & \\
\hline & Yes & 9 (20.93\%) & $40(8.87 \%)$ & $\begin{array}{l}0.37 \\
(0.16- \\
0.82)\end{array}$ & 0.015 & $\begin{array}{l}0.15 \\
(0.03- \\
0.83)\end{array}$ & 1 \\
\hline \multirow[t]{2}{*}{ Coryza; n (\%) } & No & $\begin{array}{l}28 \\
(65.12 \%)\end{array}$ & $\begin{array}{l}415 \\
(92.02 \%)\end{array}$ & Reference & & & \\
\hline & Yes & $\begin{array}{l}15 \\
(34.88 \%)\end{array}$ & $36(7.98 \%)$ & $\begin{array}{l}0.16 \\
(0.08- \\
0.33)\end{array}$ & $<0.001$ & $\begin{array}{l}0.55 \\
(0.15- \\
2.11)\end{array}$ & 1 \\
\hline \multirow[t]{2}{*}{$\begin{array}{l}\text { Dyspneia; n } \\
(\%)\end{array}$} & No & 35 (81.4\%) & $\begin{array}{l}139 \\
(30.82 \%)\end{array}$ & Reference & & & \\
\hline & Yes & $8(18.6 \%)$ & $\begin{array}{l}312 \\
(69.18 \%)\end{array}$ & $\begin{array}{l}9.82 \\
(4.44- \\
21.72)\end{array}$ & $<0.001$ & $\begin{array}{l}8.12 \\
(2.28- \\
28.96)\end{array}$ & 0.05 \\
\hline \multirow[t]{2}{*}{$\begin{array}{l}\text { Odynophagy; } \\
\text { n (\%) }\end{array}$} & No & $\begin{array}{l}27 \\
(62.79 \%)\end{array}$ & $\begin{array}{l}435 \\
(96.45 \%)\end{array}$ & Reference & & & \\
\hline & Yes & $\begin{array}{l}16 \\
(37.21 \%)\end{array}$ & 16 (3.55\%) & $\begin{array}{l}0.06 \\
(0.03- \\
0.14)\end{array}$ & $<0.001$ & $\begin{array}{l}0.25 \\
(0.06- \\
1.07)\end{array}$ & 1 \\
\hline \multirow[t]{2}{*}{$\begin{array}{l}\text { Anosmia; n } \\
(\%)\end{array}$} & Yes & $\begin{array}{l}28 \\
(65.12 \%)\end{array}$ & 48 (10.64\%) & Reference & & & \\
\hline & No & $\begin{array}{l}15 \\
(34.88 \%)\end{array}$ & $\begin{array}{l}403 \\
(89.36 \%)\end{array}$ & $\begin{array}{l}15.67 \\
(7.82- \\
31.4)\end{array}$ & $<0.001$ & $\begin{array}{l}6.86(2- \\
23.54)\end{array}$ & 0.089 \\
\hline \multirow[t]{2}{*}{$\begin{array}{l}\text { Loss Of } \\
\text { Taste; } \mathrm{n}(\%)\end{array}$} & Yes & $\begin{array}{l}25 \\
(58.14 \%)\end{array}$ & $40(8.87 \%)$ & Reference & & & \\
\hline & No & $\begin{array}{l}18 \\
(41.86 \%)\end{array}$ & $\begin{array}{l}411 \\
(91.13 \%)\end{array}$ & $\begin{array}{l}14.27 \\
(7.18- \\
28.38)\end{array}$ & $<0.001$ & $\begin{array}{l}6.62 \\
(1.94- \\
22.51)\end{array}$ & 0.1 \\
\hline $\begin{array}{l}\text { Diarrhea; n } \\
(\%)\end{array}$ & No & $\begin{array}{l}31 \\
(72.09 \%)\end{array}$ & $\begin{array}{l}411 \\
(91.13 \%)\end{array}$ & Reference & & & \\
\hline
\end{tabular}

${ }^{a}$ Odds ratios were adjusted by skin color, schooling, gender, age, and associated comorbidities such as diabetes mellitus and coronary artery disease. ${ }^{\mathrm{b}} P$-values were calculated using the unconditional logistic regression model. Associations were considered significant with a value of * $P<0.05$. N: number of individuals in each group; aOR: adjusted odds ratio; $95 \% \mathrm{Cl}$ : $95 \%$ confidence interval; NC: not calculated. 


\begin{tabular}{|c|c|c|c|c|c|c|c|}
\hline \multirow[t]{3}{*}{ Features } & & \multicolumn{2}{|l|}{ Outcomes } & \multirow{3}{*}{$\begin{array}{l}\text { OR } \\
\text { (CI95\%) }\end{array}$} & \multirow{3}{*}{$\begin{array}{l}P \\
\text { value }^{\mathrm{b}}\end{array}$} & \multirow{3}{*}{$\begin{array}{l}\mathrm{aOR}^{\mathrm{a}} \\
(\mathrm{Cl} 95 \%)\end{array}$} & \multirow{3}{*}{$\begin{array}{l}\text { Adjusted } \\
P_{\text {-value }}\end{array}$} \\
\hline & & \multirow{2}{*}{$\begin{array}{l}\text { Mild group } \\
\mathrm{N}=43\end{array}$} & \multirow{2}{*}{$\begin{array}{l}\text { Hospitalized } \\
\text { group } \\
\mathrm{N}=451\end{array}$} & & & & \\
\hline & & & & & & & \\
\hline & Yes & $\begin{array}{l}12 \\
(27.91 \%)\end{array}$ & 40 (8.87\%) & $\begin{array}{l}0.25 \\
(0.12- \\
0.53)\end{array}$ & $<0.001$ & $\begin{array}{l}0.1 \\
(0.02- \\
0.49)\end{array}$ & 0.18 \\
\hline \multirow[t]{2}{*}{$\begin{array}{l}\text { Abdominal } \\
\text { Pain; n (\%) }\end{array}$} & No & 35 (81.4\%) & $\begin{array}{l}438 \\
(97.12 \%)\end{array}$ & \multicolumn{4}{|l|}{ Reference } \\
\hline & Yes & $8(18.6 \%)$ & $13(2.88 \%)$ & $\begin{array}{l}0.13 \\
(0.05- \\
0.33)\end{array}$ & $<0.001$ & $\begin{array}{l}0.03(0- \\
0.25)\end{array}$ & 0.059 \\
\hline \multirow[t]{2}{*}{$\begin{array}{l}\text { Nausea; n } \\
(\%)\end{array}$} & No & $\begin{array}{l}34 \\
(79.07 \%)\end{array}$ & $\begin{array}{l}427 \\
(94.68 \%)\end{array}$ & \multicolumn{4}{|l|}{ Reference } \\
\hline & Yes & $9(20.93 \%)$ & $24(5.32 \%)$ & $\begin{array}{l}0.21 \\
(0.09- \\
0.49)\end{array}$ & $<0.001$ & $\begin{array}{l}0.03(0- \\
0.26)\end{array}$ & 0.046 \\
\hline \multirow[t]{2}{*}{$\begin{array}{l}\text { Headache; } n \\
(\%)\end{array}$} & Yes & $\begin{array}{l}26 \\
(60.47 \%)\end{array}$ & 70 (15.52\%) & \multicolumn{4}{|l|}{ Reference } \\
\hline & No & $\begin{array}{l}17 \\
(39.53 \%)\end{array}$ & $\begin{array}{l}381 \\
(84.48 \%)\end{array}$ & $\begin{array}{l}8.32 \\
(4.29- \\
16.14)\end{array}$ & $<0.001$ & $\begin{array}{l}6.34 \\
(1.84- \\
21.91)\end{array}$ & 0.14 \\
\hline \multirow[t]{2}{*}{$\begin{array}{l}\text { Myalgia; n } \\
(\%)\end{array}$} & Yes & $\begin{array}{l}26 \\
(60.47 \%)\end{array}$ & 97 (21.51\%) & \multicolumn{4}{|l|}{ Reference } \\
\hline & No & $\begin{array}{l}17 \\
(39.53 \%)\end{array}$ & $\begin{array}{l}354 \\
(78.49 \%)\end{array}$ & $\begin{array}{l}5.58 \\
(2.91- \\
10.71)\end{array}$ & $<0.001$ & $\begin{array}{l}4.12 \\
(1.25- \\
13.57)\end{array}$ & 0.795 \\
\hline \multicolumn{8}{|c|}{$\begin{array}{l}\text { a'Odds ratios were adjusted by skin color, schooling, gender, age, and associated comorbidities such } \\
\text { as diabetes mellitus and coronary artery disease. }{ }^{b} P \text {-values were calculated using the unconditional } \\
\text { logistic regression model. Associations were considered significant with a value of * } P<0.05 \text {. N: } \\
\text { number of individuals in each group; aOR: adjusted odds ratio; } 95 \% \mathrm{Cl}: 95 \% \text { confidence interval; NC: } \\
\text { not calculated. }\end{array}$} \\
\hline
\end{tabular}

\section{Alleles, genotypes, and haplotype of inflammasome genes and risk for hospitalization}

पThe genotypes, alleles, and carrier frequencies of all the studied SNP associated with hospitalization are shown in Table 2. Genotype frequencies associated with the 11 SNPs analyzed were in Hardy-Weinberg equilibrium in both groups. 
Table 2

Unconditional logistic multiple regression model of risk and protection genetic factors for hospitalization in SARS-CoV-2 infected individuals ( $\mathrm{n}=494)$.

\begin{tabular}{|c|c|c|c|c|c|}
\hline \multirow{3}{*}{$\begin{array}{l}\text { Genes } \\
\text { SNP (rs) }\end{array}$} & \multirow{3}{*}{$\begin{array}{l}\text { Alleles and } \\
\text { Genotypes }\end{array}$} & \multirow{3}{*}{$\begin{array}{l}\text { Hospitalized } \\
\text { group } \\
\mathrm{N}=451 \\
\mathrm{~N}(\%)\end{array}$} & \multirow{3}{*}{$\begin{array}{l}\text { Mild } \\
\text { group } \\
\mathrm{N}=43 \\
\mathrm{~N}(\%)\end{array}$} & \multirow{3}{*}{$\begin{array}{l}\text { aOR }^{a} \\
\text { (Cl 95\%) }\end{array}$} & \multirow[t]{3}{*}{$\begin{array}{l}P \\
\text { value }^{\text {b }}\end{array}$} \\
\hline & & & & & \\
\hline & & & & & \\
\hline \multirow[t]{9}{*}{$\begin{array}{l}\text { CARD8 } \\
\text { rs2043211 }\end{array}$} & $A / A$ & 248 (54.99) & $\begin{array}{l}22 \\
(51.16)\end{array}$ & Reference & \\
\hline & $A / T$ & $169(37.47)$ & $\begin{array}{l}16 \\
(37.21)\end{array}$ & $0.92(0.28-3.03)$ & 0.89 \\
\hline & $\mathrm{T} / \mathrm{T}$ & $34(7.54)$ & $5(11.63)$ & $1.42(0.15-13.39)$ & 0.759 \\
\hline & $A$ & $665(73.73)$ & $\begin{array}{l}60 \\
(69.77)\end{array}$ & Reference & \\
\hline & $\mathrm{T}$ & $237(26.27)$ & $\begin{array}{l}26 \\
(30.23)\end{array}$ & $0.98(0.94-1.02)$ & 0.247 \\
\hline & $\begin{array}{l}\text { Non-Carrier- } \\
\text { A }\end{array}$ & $34(7.54)$ & $5(11.63)$ & Reference & \\
\hline & Carrier-A & $417(92.46)$ & $\begin{array}{l}38 \\
(88.37)\end{array}$ & $0.68(0.08-6.22)$ & 0.735 \\
\hline & $\begin{array}{l}\text { Non Carrier- } \\
T_{T}\end{array}$ & 248 (54.99) & $\begin{array}{l}22 \\
(51.16)\end{array}$ & Reference & \\
\hline & Carrier-T & $203(45.01)$ & $\begin{array}{l}21 \\
(48.84)\end{array}$ & $0.99(0.32-3.05)$ & 0.984 \\
\hline \multirow{3}{*}{$\begin{array}{l}\text { CARD8 } \\
\text { rs6509365 }\end{array}$} & $A / A$ & $222(49.22)$ & $\begin{array}{l}22 \\
(51.16)\end{array}$ & Reference & \\
\hline & $A / G$ & $184(40.8)$ & $\begin{array}{l}16 \\
(37.21)\end{array}$ & $1.02(0.31-3.36)$ & 0.971 \\
\hline & $\mathrm{G} / \mathrm{G}$ & $45(9.98)$ & $5(11.63)$ & $1.63(0.19-13.92)$ & 0.653 \\
\hline
\end{tabular}

aOdds ratios were adjusted by skin color, schooling, gender, age, and associated comorbidities, such as diabetes mellitus and coronary artery disease. ${ }^{\mathrm{b}} P$-values were calculated using the unconditional logistic regression model. Associations were considered significant with a value of $* P<0.05$. ${ }^{\mathrm{C}}$ The rs1143634 polymorphism in the IL-1 $\beta$ gene determination was not possible for one individual from the hospitalized group. N: number of individuals in each group; aOR: adjusted odds ratio; $95 \% \mathrm{Cl}: 95 \%$ confidence interval; NC: not calculated; $\mathrm{A}, \mathrm{T}, \mathrm{G}$, and $\mathrm{C}=$ each allele count, irrespective of the genotype. Carrier-A = total of genotypes with the A allele; Carrier-T = total of genotypes with $\mathrm{T}$ allele; Carrier-C = total of genotypes with the $\mathrm{C}$ allele; Carrier- $\mathrm{G}=$ total of genotypes with the $\mathrm{G}$ allele; Non-Carrier- $\mathrm{A}=$ total of genotypes without the A allele; Non-Carrier- $T$ = total of genotypes without the T allele; NonCarrier- $C=$ total of genotypes without the $C$ allele; Non-Carrier- $G=$ total of genotypes without the $G$ allele. 


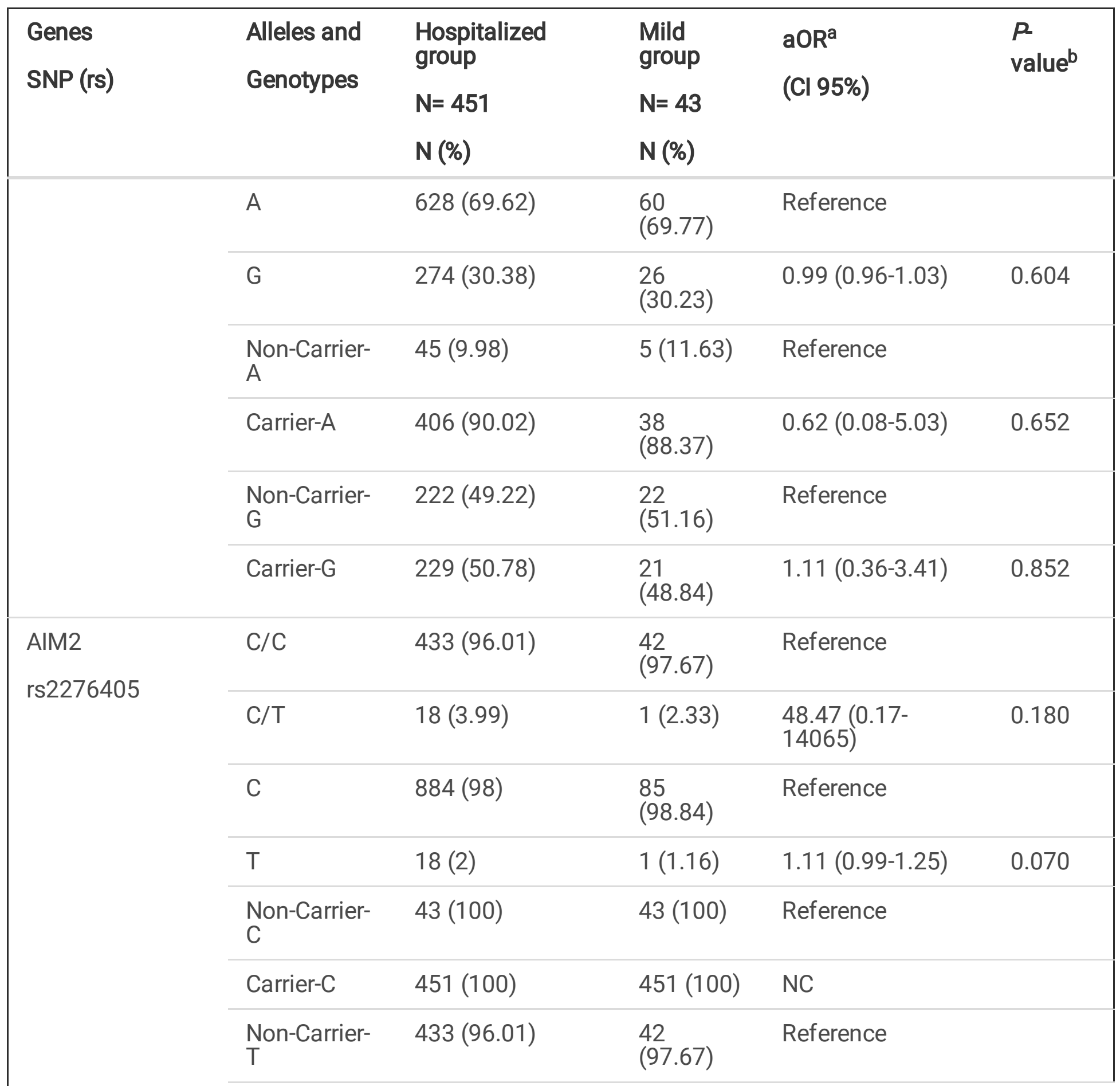

aOdds ratios were adjusted by skin color, schooling, gender, age, and associated comorbidities, such as diabetes mellitus and coronary artery disease. ${ }^{\mathrm{b}} P$-values were calculated using the unconditional logistic regression model. Associations were considered significant with a value of $* P<0.05$. ${ }^{\mathrm{C}}$ The rs1143634 polymorphism in the IL-1 $\beta$ gene determination was not possible for one individual from the hospitalized group. N: number of individuals in each group; aOR: adjusted odds ratio; $95 \% \mathrm{Cl}$ : $95 \%$ confidence interval; NC: not calculated; $\mathrm{A}, \mathrm{T}, \mathrm{G}$, and $\mathrm{C}=$ each allele count, irrespective of the genotype. Carrier-A = total of genotypes with the A allele; Carrier-T = total of genotypes with T allele; Carrier-C = total of genotypes with the $\mathrm{C}$ allele; Carrier- $\mathrm{G}=$ total of genotypes with the $\mathrm{G}$ allele; Non-Carrier- $\mathrm{A}=$ total of genotypes without the A allele; Non-Carrier-T = total of genotypes without the T allele; NonCarrier- $C=$ total of genotypes without the $C$ allele; Non-Carrier- $G=$ total of genotypes without the $G$ allele. 


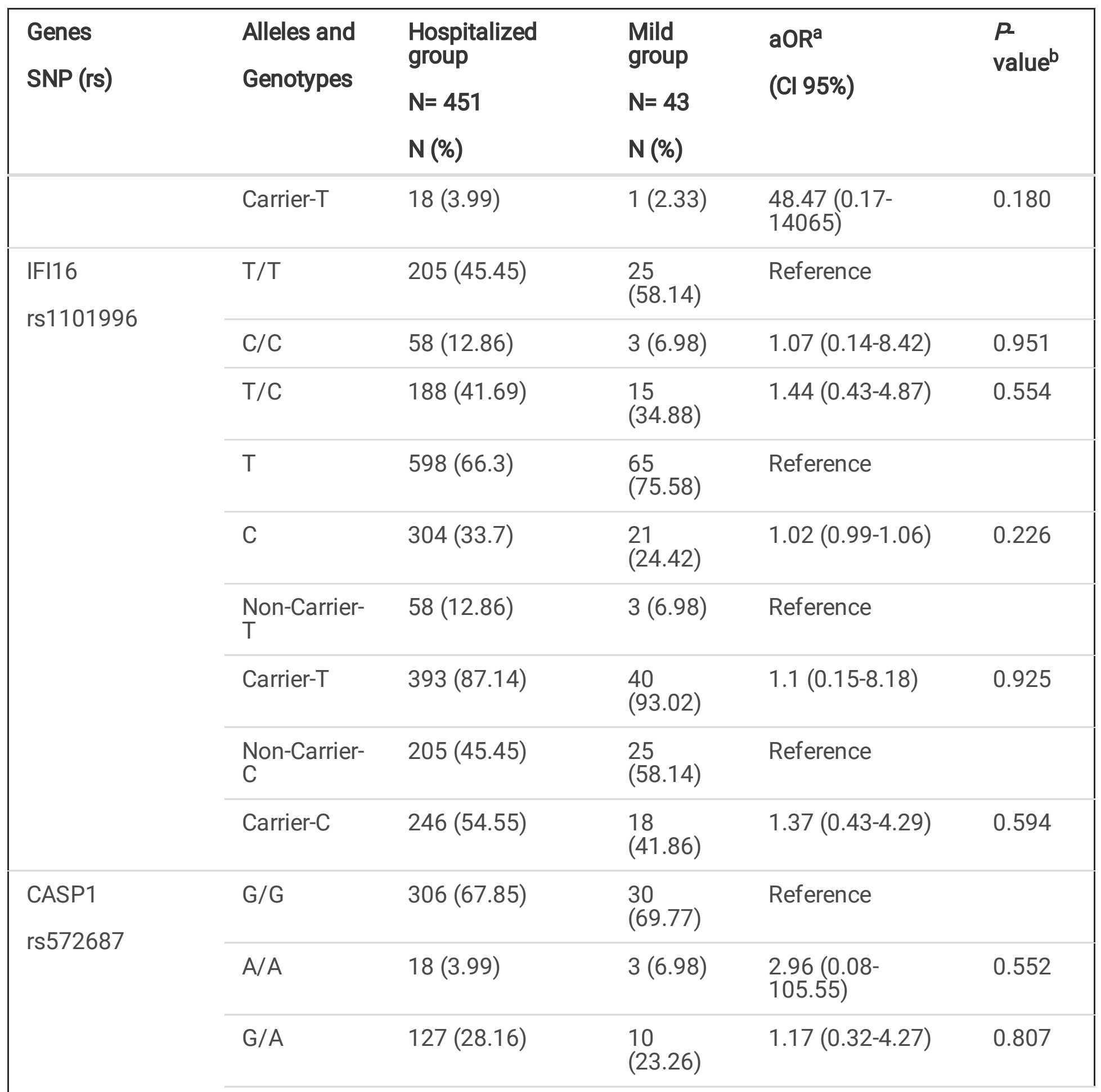

aOdds ratios were adjusted by skin color, schooling, gender, age, and associated comorbidities, such as diabetes mellitus and coronary artery disease. ${ }^{\mathrm{b}} P$-values were calculated using the unconditional logistic regression model. Associations were considered significant with a value of $* P<0.05$. ${ }^{\mathrm{C}}$ The rs1143634 polymorphism in the IL-1 $\beta$ gene determination was not possible for one individual from the hospitalized group. N: number of individuals in each group; aOR: adjusted odds ratio; $95 \% \mathrm{Cl}: 95 \%$ confidence interval; NC: not calculated; $\mathrm{A}, \mathrm{T}, \mathrm{G}$, and $\mathrm{C}=$ each allele count, irrespective of the genotype. Carrier- $\mathrm{A}=$ total of genotypes with the $\mathrm{A}$ allele; Carrier-T = total of genotypes with $\mathrm{T}$ allele; Carrier-C = total of genotypes with the $\mathrm{C}$ allele; Carrier- $\mathrm{G}=$ total of genotypes with the $\mathrm{G}$ allele; Non-Carrier- $\mathrm{A}=$ total of genotypes without the A allele; Non-Carrier-T = total of genotypes without the T allele; NonCarrier- $C=$ total of genotypes without the $C$ allele; Non-Carrier $-G=$ total of genotypes without the $G$ allele. 


\begin{tabular}{|c|c|c|c|c|c|}
\hline \multirow[t]{3}{*}{$\begin{array}{l}\text { Genes } \\
\text { SNP (rs) }\end{array}$} & \multirow[t]{3}{*}{$\begin{array}{l}\text { Alleles and } \\
\text { Genotypes }\end{array}$} & \multirow{3}{*}{$\begin{array}{l}\text { Hospitalized } \\
\text { group } \\
\mathrm{N}=451 \\
\mathrm{~N}(\%)\end{array}$} & \multirow{3}{*}{$\begin{array}{l}\begin{array}{l}\text { Mild } \\
\text { group }\end{array} \\
\mathrm{N}=43 \\
\mathrm{~N}(\%)\end{array}$} & \multirow[t]{3}{*}{$\begin{array}{l}\text { aOR }^{a} \\
\text { (Cl 95\%) }\end{array}$} & \multirow[t]{3}{*}{$\begin{array}{l}P \\
\text { value }^{b}\end{array}$} \\
\hline & & & & & \\
\hline & & & & & \\
\hline & G & 739 (81.93) & 70 (81.4) & Reference & \\
\hline & $A$ & 163 (18.07) & 16 (18.6) & $1.01(0.97-1.05)$ & 0.639 \\
\hline & $\begin{array}{l}\text { Non-Carrier- } \\
\mathrm{G}\end{array}$ & 18 (3.99) & $3(6.98)$ & Reference & \\
\hline & Carrier-G & 433 (96.01) & $\begin{array}{l}40 \\
(93.02)\end{array}$ & $0.36(0.01-12.6)$ & 0.571 \\
\hline & $\begin{array}{l}\text { Non-Carrier- } \\
\text { A }\end{array}$ & $306(67.85)$ & $\begin{array}{l}30 \\
(69.77)\end{array}$ & Reference & \\
\hline & Carrier-A & $145(32.15)$ & $\begin{array}{l}13 \\
(30.23)\end{array}$ & $1.27(0.36-4.48)$ & 0.705 \\
\hline \multirow{8}{*}{$\begin{array}{l}\text { IL-1 } \\
\text { rs1143634c }\end{array}$} & $\mathrm{G} / \mathrm{G}$ & $292(64.75)$ & $\begin{array}{l}22 \\
(51.16)\end{array}$ & Reference & \\
\hline & $\mathrm{A} / \mathrm{A}$ & $12(2.66)$ & $1(2.33)$ & $0.18(0-13.45)$ & 0.432 \\
\hline & $\mathrm{G} / \mathrm{A}$ & 146 (32.37) & $\begin{array}{l}20 \\
(46.51)\end{array}$ & $0.58(0.17-2.04)$ & 0.399 \\
\hline & G & 730 (80.93) & $\begin{array}{l}64 \\
(74.42)\end{array}$ & Reference & \\
\hline & $A$ & $170(18.85)$ & $\begin{array}{l}22 \\
(25.58)\end{array}$ & $0.97(0.93-1.01)$ & 0.201 \\
\hline & $\begin{array}{l}\text { Non-Carrier- } \\
G\end{array}$ & $13(2.88)$ & $1(2.33)$ & Reference & \\
\hline & Carrier-G & 438 (97.12) & $\begin{array}{l}42 \\
(97.67)\end{array}$ & $\begin{array}{l}4.91(0.06- \\
410.47)\end{array}$ & 0.481 \\
\hline & $\begin{array}{l}\text { Non-Carrier- } \\
\text { A }\end{array}$ & $293(64.97)$ & $\begin{array}{l}22 \\
(51.16)\end{array}$ & Reference & \\
\hline
\end{tabular}

aOdds ratios were adjusted by skin color, schooling, gender, age, and associated comorbidities, such as diabetes mellitus and coronary artery disease. ${ }^{\mathrm{b}} P$-values were calculated using the unconditional logistic regression model. Associations were considered significant with a value of $* P<0.05$. ${ }^{\mathrm{C}}$ The rs1143634 polymorphism in the IL-1 $\beta$ gene determination was not possible for one individual from the hospitalized group. N: number of individuals in each group; aOR: adjusted odds ratio; $95 \% \mathrm{Cl}$ : $95 \%$ confidence interval; NC: not calculated; $\mathrm{A}, \mathrm{T}, \mathrm{G}$, and $\mathrm{C}=$ each allele count, irrespective of the genotype. Carrier-A = total of genotypes with the A allele; Carrier-T = total of genotypes with T allele; Carrier-C = total of genotypes with the $\mathrm{C}$ allele; Carrier- $\mathrm{G}=$ total of genotypes with the $\mathrm{G}$ allele; Non-Carrier- $\mathrm{A}=$ total of genotypes without the A allele; Non-Carrier-T = total of genotypes without the T allele; NonCarrier- $C=$ total of genotypes without the $C$ allele; Non-Carrier $-G=$ total of genotypes without the $G$ allele. 


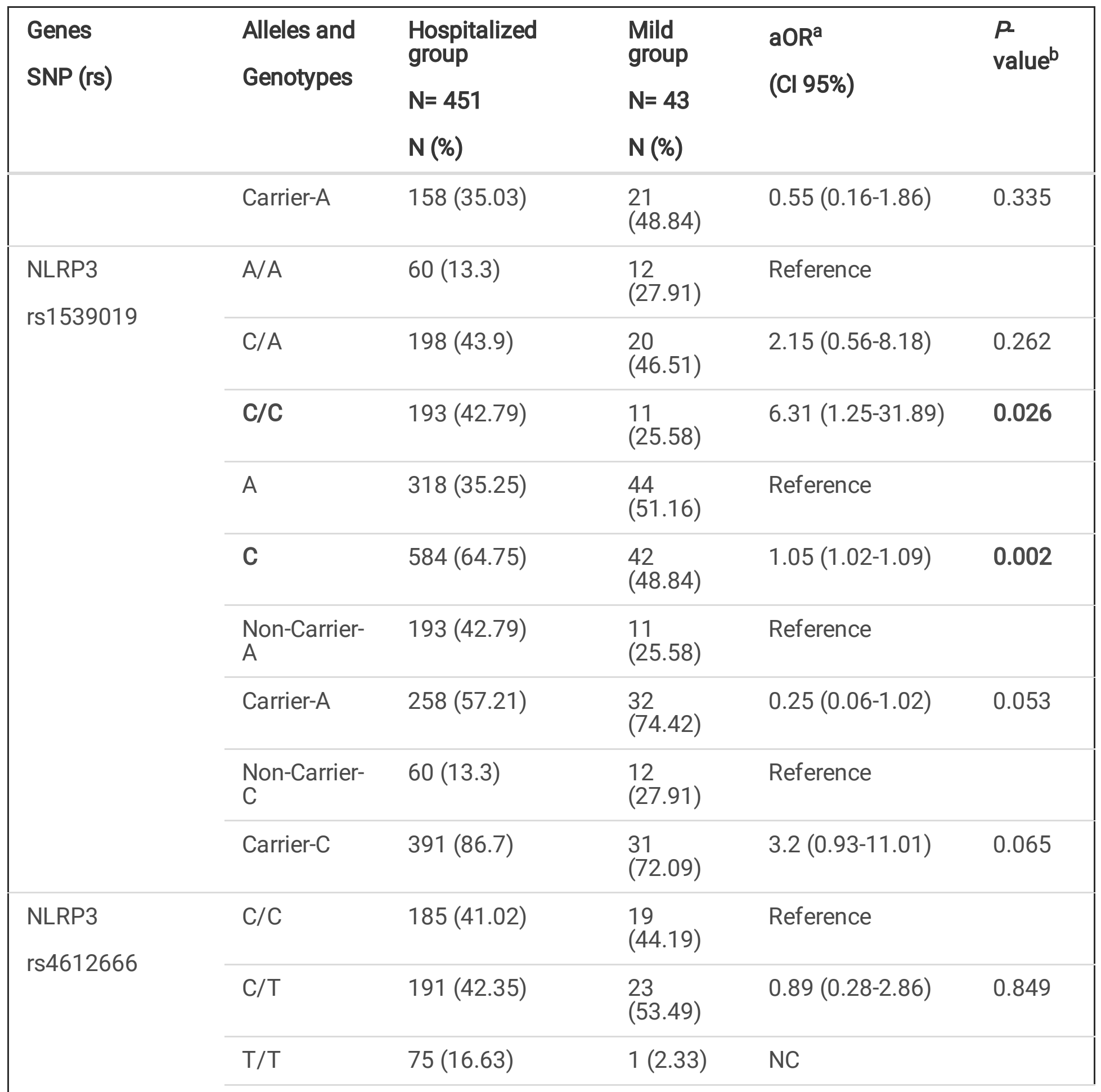

aOdds ratios were adjusted by skin color, schooling, gender, age, and associated comorbidities, such as diabetes mellitus and coronary artery disease. ${ }^{\mathrm{b}} P$-values were calculated using the unconditional logistic regression model. Associations were considered significant with a value of $* P<0.05$. ${ }^{\mathrm{C}}$ The rs1143634 polymorphism in the IL-1 $\beta$ gene determination was not possible for one individual from the hospitalized group. N: number of individuals in each group; aOR: adjusted odds ratio; $95 \% \mathrm{Cl}: 95 \%$ confidence interval; NC: not calculated; $\mathrm{A}, \mathrm{T}, \mathrm{G}$, and $\mathrm{C}=$ each allele count, irrespective of the genotype. Carrier-A = total of genotypes with the A allele; Carrier-T = total of genotypes with T allele; Carrier-C = total of genotypes with the $\mathrm{C}$ allele; Carrier- $\mathrm{G}=$ total of genotypes with the $\mathrm{G}$ allele; Non-Carrier- $\mathrm{A}=$ total of genotypes without the A allele; Non-Carrier-T = total of genotypes without the T allele; NonCarrier- $C=$ total of genotypes without the $C$ allele; Non-Carrier $-G=$ total of genotypes without the $G$ allele. 


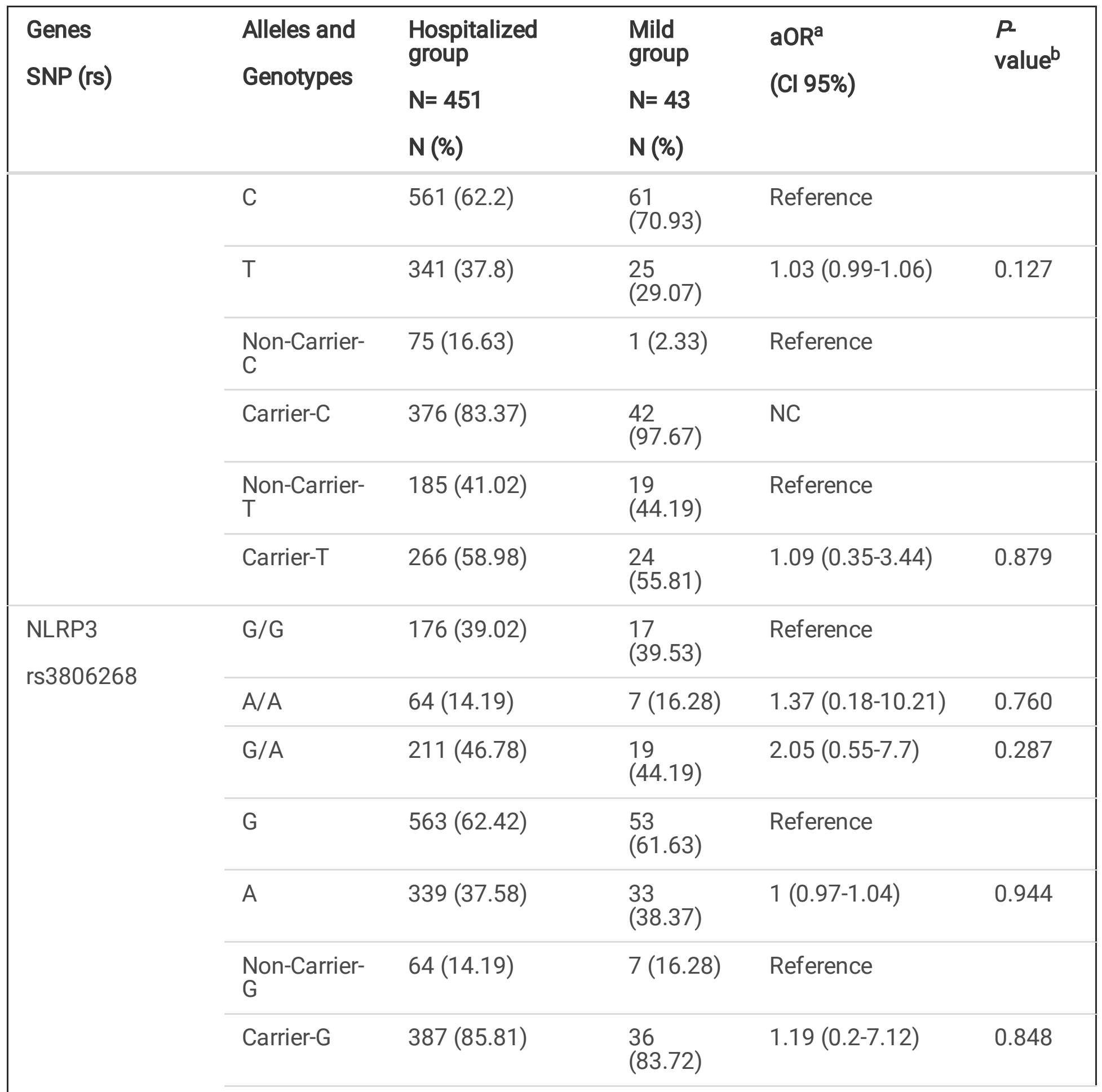

aOdds ratios were adjusted by skin color, schooling, gender, age, and associated comorbidities, such as diabetes mellitus and coronary artery disease. ${ }^{\mathrm{b}} P$-values were calculated using the unconditional logistic regression model. Associations were considered significant with a value of $* P<0.05$. ${ }^{\mathrm{C}}$ The rs1143634 polymorphism in the IL-1 $\beta$ gene determination was not possible for one individual from the hospitalized group. N: number of individuals in each group; aOR: adjusted odds ratio; $95 \% \mathrm{Cl}: 95 \%$ confidence interval; NC: not calculated; $\mathrm{A}, \mathrm{T}, \mathrm{G}$, and $\mathrm{C}=$ each allele count, irrespective of the genotype. Carrier- $\mathrm{A}=$ total of genotypes with the $\mathrm{A}$ allele; Carrier-T = total of genotypes with $\mathrm{T}$ allele; Carrier-C = total of genotypes with the $\mathrm{C}$ allele; Carrier- $\mathrm{G}=$ total of genotypes with the $\mathrm{G}$ allele; Non-Carrier- $\mathrm{A}=$ total of genotypes without the A allele; Non-Carrier-T = total of genotypes without the T allele; NonCarrier- $C=$ total of genotypes without the $C$ allele; Non-Carrier $-G=$ total of genotypes without the $G$ allele. 


\begin{tabular}{|c|c|c|c|c|c|}
\hline \multirow[t]{5}{*}{$\begin{array}{l}\text { Genes } \\
\text { SNP (rs) }\end{array}$} & \multirow[t]{3}{*}{$\begin{array}{l}\text { Alleles and } \\
\text { Genotypes }\end{array}$} & $\begin{array}{l}\text { Hospitalized } \\
\text { group }\end{array}$ & $\begin{array}{l}\text { Mild } \\
\text { group }\end{array}$ & \multirow[t]{3}{*}{$\begin{array}{l}a_{0 R}^{a} \\
(C l 95 \%)\end{array}$} & \multirow[t]{3}{*}{$\begin{array}{l}P \\
\text { value }^{b}\end{array}$} \\
\hline & & IV & $10-40$ & & \\
\hline & & $\mathbf{N}(\%)$ & $\mathbf{N}(\%)$ & & \\
\hline & $\begin{array}{l}\text { Non-Carrier- } \\
\text { A }\end{array}$ & 176 (39.02) & $\begin{array}{l}17 \\
(39.53)\end{array}$ & Reference & \\
\hline & Carrier-A & 275 (60.98) & $\begin{array}{l}26 \\
(60.47)\end{array}$ & $1.92(0.53-6.96)$ & 0.319 \\
\hline \multirow{9}{*}{$\begin{array}{l}\text { NLRP3 } \\
\text { rs35829419 }\end{array}$} & $\mathrm{C} / \mathrm{C}$ & 432 (95.79) & $\begin{array}{l}42 \\
(97.67)\end{array}$ & Reference & \\
\hline & $\mathrm{A} / \mathrm{A}$ & $1(0.22)$ & $0(0)$ & $\mathrm{NC}$ & \\
\hline & $\mathrm{C} / \mathrm{A}$ & $18(3.99)$ & $1(2.33)$ & $0.52(0.04-6.67)$ & 0.614 \\
\hline & C & 882 (97.78) & $\begin{array}{l}85 \\
(98.84)\end{array}$ & Reference & \\
\hline & A & $20(2.22)$ & $1(1.16)$ & $1.02(0.92-1.14)$ & 0.642 \\
\hline & $\begin{array}{l}\text { Non-Carrier- } \\
\text { C }\end{array}$ & $1(0.22)$ & $0(0)$ & Reference & \\
\hline & Carrier-C & 450 (99.78) & $43(100)$ & $\mathrm{NC}$ & \\
\hline & $\begin{array}{l}\text { Non-Carrier- } \\
\text { A }\end{array}$ & 432 (95.79) & $\begin{array}{l}42 \\
(97.67)\end{array}$ & Reference & \\
\hline & Carrier-A & $19(4.21)$ & $1(2.33)$ & $0.52(0.04-6.67)$ & 0.614 \\
\hline \multirow{4}{*}{$\begin{array}{l}\text { NLRP3 } \\
\text { rs10754558 }\end{array}$} & $\mathrm{C} / \mathrm{C}$ & $196(43.46)$ & $\begin{array}{l}11 \\
(25.58)\end{array}$ & Reference & \\
\hline & $C / G$ & $201(44.57)$ & $\begin{array}{l}27 \\
(62.79)\end{array}$ & $0.16(0.04-0.71)$ & 0.016 \\
\hline & $\mathrm{G} / \mathrm{G}$ & $54(11.97)$ & $5(11.63)$ & $0.4(0.06-2.45)$ & 0.319 \\
\hline & C & $593(65.74)$ & $\begin{array}{l}49 \\
(56.98)\end{array}$ & Reference & \\
\hline
\end{tabular}

aOdds ratios were adjusted by skin color, schooling, gender, age, and associated comorbidities, such as diabetes mellitus and coronary artery disease. ${ }^{\mathrm{b}} P$-values were calculated using the unconditional logistic regression model. Associations were considered significant with a value of $* P<0.05$. ${ }^{\mathrm{C}}$ The rs1143634 polymorphism in the IL-1 $\beta$ gene determination was not possible for one individual from the hospitalized group. N: number of individuals in each group; aOR: adjusted odds ratio; $95 \% \mathrm{Cl}: 95 \%$ confidence interval; NC: not calculated; $\mathrm{A}, \mathrm{T}, \mathrm{G}$, and $\mathrm{C}=$ each allele count, irrespective of the genotype. Carrier- $\mathrm{A}=$ total of genotypes with the $\mathrm{A}$ allele; Carrier-T = total of genotypes with $\mathrm{T}$ allele; Carrier-C = total of genotypes with the $\mathrm{C}$ allele; Carrier- $\mathrm{G}=$ total of genotypes with the $\mathrm{G}$ allele; Non-Carrier- $\mathrm{A}=$ total of genotypes without the A allele; Non-Carrier- $T$ = total of genotypes without the T allele; NonCarrier- $C=$ total of genotypes without the $C$ allele; Non-Carrier $-G=$ total of genotypes without the $G$ allele. 


\begin{tabular}{|c|c|c|c|c|c|}
\hline \multirow[t]{6}{*}{$\begin{array}{l}\text { Genes } \\
\text { SNP (rs) }\end{array}$} & $\begin{array}{l}\text { Alleles and } \\
\text { Genotypes }\end{array}$ & $\begin{array}{l}\text { Hospitalized } \\
\text { group } \\
\mathrm{N}=451 \\
\mathrm{~N}(\%)\end{array}$ & $\begin{array}{l}\begin{array}{l}\text { Mild } \\
\text { group }\end{array} \\
N=43 \\
N(\%)\end{array}$ & $\begin{array}{l}\text { aOR }^{a} \\
\text { (Cl 95\%) }\end{array}$ & $\begin{array}{l}P \\
\text { value }^{b}\end{array}$ \\
\hline & G & 309 (34.26) & $\begin{array}{l}37 \\
(43.02)\end{array}$ & $0.97(0.94-1.01)$ & 0.106 \\
\hline & $\begin{array}{l}\text { Non-Carrier- } \\
\mathrm{C}\end{array}$ & 54 (11.97) & $5(11.63)$ & Reference & \\
\hline & Carrier-C & 397 (88.03) & $\begin{array}{l}38 \\
(88.37)\end{array}$ & $0.74(0.17-3.19)$ & 0.688 \\
\hline & $\begin{array}{l}\text { Non-Carrier- } \\
\mathrm{G}\end{array}$ & $196(43.46)$ & $\begin{array}{l}11 \\
(25.58)\end{array}$ & Reference & \\
\hline & Carrier-G & $255(56.54)$ & $\begin{array}{l}32 \\
(74.42)\end{array}$ & $0.2(0.05-0.84)$ & 0.028 \\
\hline \multicolumn{6}{|c|}{ 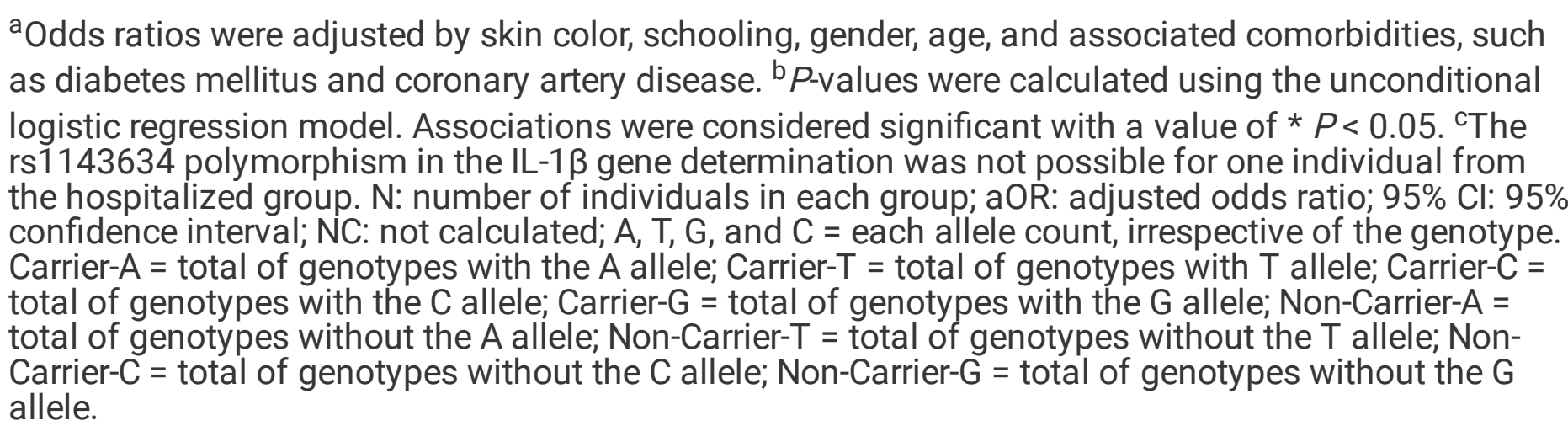 } \\
\hline
\end{tabular}

In this study we observed that carrying the $\mathrm{C} / \mathrm{C}$ genotype $\left(\mathrm{OR}_{\mathrm{adj}}=6.31(95 \% \mathrm{Cl}, 1.25-31.89), P_{\mathrm{adj}}=0.026\right)$ or the allele $\mathrm{C}\left(\mathrm{OR}_{\mathrm{adj}}=1.05(95 \% \mathrm{Cl}, 1.02-1.09), P_{\mathrm{adj}}=0.002\right)$ in the NLRP3 variant rs1539019 was associated with the risk for hospitalization in SARS-CoV-2 infected individuals (Table 2). The frequency of carrier-C in this variant was slightly higher among the Hospitalized group (86.7\%) compared with the Mild group (72.1\%), constituting a genetic marker with a trend for the risk of hospitalization in SARS-CoV2 infected individuals $\left(\mathrm{OR}_{\mathrm{adj}}=3.2(95 \% \mathrm{Cl}, 0.93-11.01), P_{\mathrm{adj}}=0.065\right)$. On the other hand, the frequency of carrier-A in this variant was slightly higher among the Mild group (74.4\%) compared with the Hospitalized group (57.2\%), constituting a genetic marker with a trend for the protection against hospitalization in SARS-CoV-2 infected individuals $\left(\mathrm{OR}_{\mathrm{adj}}=0.25(95 \% \mathrm{Cl}, 0.06-1.02), P_{\mathrm{adj}}=0.053\right)$.

Furthermore, the $\mathrm{C} / \mathrm{G}$ genotype $\left(\mathrm{OR}_{\mathrm{adj}}=0.16(95 \% \mathrm{Cl}, 0.04-0.71), P_{\mathrm{adj}}=0.016\right)$ or carrier-G $\left(\mathrm{OR}_{\mathrm{adj}}=0.2(95 \%\right.$ $\left.\mathrm{Cl}, 0.05-0.84), P_{\mathrm{adj}}=0.028\right)$ in NLRP3 variant $\mathrm{rs} 10754558$ was associated with protection for hospitalization in SARS-CoV-2 infected individuals (Table 2). The frequency of the T allele in AIM2 variant rs2276405 was also slightly different between the Mild group (1.16\%) and the hospitalized group (2\%) $\left(\mathrm{OR}_{\mathrm{adj}}=1.11(95 \% \mathrm{Cl}, 0.99-1.25), P_{\mathrm{adj}}=0.070\right)$. The SNPs in the CARD8 (rs6509365 and rs2043211), IFI16 
(rs1101996), CASP-1 (rs572687), IL-1 (rs1143634), and NLRP3 (rs4612666, rs3806268 and rs35829419) did not reveal any significant associations with risk and/or protection for hospitalization in SARS-CoV-2 infected individuals.

With respect to haplotypes of the NLRP3 genetic variants, the A-C-G-C-G haplotype (rs1539019 rs4612666 - rs3806268 - rs35829419 - rs10754558) was associated with the protection for hospitalization in SARS-CoV-2 infected individuals $\left(\mathrm{OR}_{\mathrm{adj}}=0.14(95 \% \mathrm{Cl}, 0.02-0.83), P_{\mathrm{adj}}=0.030\right)(\mathrm{Table}$ $3)$. No haplotype of the CARD8 genetic variants was associated with risk and/or protection against hospitalization due to COVID-19 (Table 3). These analyzes were performed considering the most frequent haplotype of the NLRP3 (C-T-G-C-C haplotype) and the CARD8 (AA) genes as references. 
Table 3

Association analyses among NLRP3 and CARD8 inflammasome haplotypes frequencies and risk/protection factors for hospitalization in SARS-CoV-2 infected individuals.

\begin{tabular}{|c|c|c|c|c|c|}
\hline \multirow[t]{3}{*}{ Genes SNP (rs) } & \multirow[t]{3}{*}{ Haplotypes } & \multirow{3}{*}{$\begin{array}{l}\text { Hospitalized } \\
\text { group } \\
N=451 \\
N(\%)\end{array}$} & \multirow{3}{*}{$\begin{array}{l}\text { Mild } \\
\text { group } \\
\mathrm{N}=43 \\
\mathrm{~N}(\%)\end{array}$} & \multicolumn{2}{|l|}{ Adjusted model } \\
\hline & & & & \multirow[t]{2}{*}{$\mathrm{aOR}^{\mathrm{a}}$ (CI95\%) } & \multirow{2}{*}{$\begin{array}{l}\mathrm{P}- \\
\text { value }^{\mathrm{b}}\end{array}$} \\
\hline & & & & & \\
\hline & CTGCC & $221(24.72)$ & $\begin{array}{l}14 \\
(16.28)\end{array}$ & Reference & \\
\hline & ACACC & $49(5.48)$ & $\begin{array}{l}6 \\
(6.98)\end{array}$ & $0.52(0.06-4.53)$ & 0.558 \\
\hline & ACACG & $145(16.22)$ & $\begin{array}{l}15 \\
(17.44)\end{array}$ & $0.4(0.09-1.82)$ & 0.234 \\
\hline & ACGCC & $20(2.24)$ & $\begin{array}{l}3 \\
(3.49)\end{array}$ & $0.19(0.02-2.31)$ & 0.193 \\
\hline & ACGCG & $36(4.03)$ & $\begin{array}{l}13 \\
(15.12)\end{array}$ & $0.14(0.02-0.83)$ & 0.030 \\
\hline \multirow{9}{*}{$\begin{array}{l}\text { NLRP3 } \\
\text { rs1539019 rs } 4612666 \\
\text { rs3806268 rs35829419 } \\
\text { rs10754558 }\end{array}$} & ATGAG & $3(0.34)$ & $\begin{array}{l}1 \\
(1.16)\end{array}$ & $0.09(0-5.74)$ & 0.254 \\
\hline & ATGCC & $58(6.49)$ & $\begin{array}{l}6 \\
(6.98)\end{array}$ & $0.28(0.03-2.34)$ & 0.242 \\
\hline & CCACC & 105 (11.74) & $\begin{array}{l}9 \\
(10.47)\end{array}$ & $1.44(0.2-10.44)$ & 0.717 \\
\hline & CCACG & $30(3.36)$ & $\begin{array}{l}1 \\
(1.16)\end{array}$ & $1.84(0.05-62.74)$ & 0.736 \\
\hline & CCGAC & $1(0.11)$ & $0(0)$ & $\mathrm{NC}$ & \\
\hline & CCGCC & $128(14.32)$ & $\begin{array}{l}10 \\
(11.63)\end{array}$ & $0.56(0.1-3.03)$ & 0.503 \\
\hline & CCGCG & $43(4.81)$ & $\begin{array}{l}4 \\
(4.65)\end{array}$ & $1.58(0.11-22.1)$ & 0.732 \\
\hline & CTACC & $5(0.56)$ & $\begin{array}{l}1 \\
(1.16)\end{array}$ & $\begin{array}{l}0.55(0- \\
99602969588474.9)\end{array}$ & 0.972 \\
\hline & CTACG & $3(0.34)$ & $\begin{array}{l}1 \\
(1.16)\end{array}$ & $3.55(0-26225.93)$ & 0.780 \\
\hline
\end{tabular}

a Odds ratios were adjusted by skin color, schooling, gender, age, and associated comorbidities, such as diabetes mellitus and coronary artery disease. ${ }^{\mathrm{b}} P$-values were calculated using the unconditional logistic regression model. Associations were considered significant with a value of * $P<0.05$. aOR: adjusted odds ratio; $95 \% \mathrm{Cl}$ : $95 \%$ confidence interval; NC: not calculated; N: number of individuals in each group. 


\begin{tabular}{|c|c|c|c|c|c|}
\hline & CTGCG & $32(3.58)$ & $\begin{array}{l}2 \\
(2.33)\end{array}$ & $0.45(0.03-7.46)$ & 0.580 \\
\hline \multirow{4}{*}{$\begin{array}{l}\text { CARD8 } \\
\text { rs2043211 } \\
\text { rs6509365 }\end{array}$} & AA & $626(69.4)$ & $\begin{array}{l}59 \\
(68.6)\end{array}$ & \multicolumn{2}{|l|}{ Reference } \\
\hline & $A G$ & $39(4.32)$ & $\begin{array}{l}1 \\
(1.16)\end{array}$ & $9.44(0.06-1495.18)$ & 0.385 \\
\hline & TA & $2(0.22)$ & $\begin{array}{l}1 \\
(1.16)\end{array}$ & $4.95(0.01-4423.44)$ & 0.645 \\
\hline & TG & $235(26.05)$ & $\begin{array}{l}25 \\
(29.07)\end{array}$ & $1.06(0.43-2.62)$ & 0.892 \\
\hline \multicolumn{6}{|c|}{$\begin{array}{l}\text { aOdds ratios were adjusted by skin color, schooling, gender, age, and associated comorbidities, such } \\
\text { as diabetes mellitus and coronary artery disease. b } P \text {-values were calculated using the unconditional } \\
\text { logistic regression model. Associations were considered significant with a value of } * P<0.05 \text {. aOR: } \\
\text { adjusted odds ratio; } 95 \% \mathrm{Cl} \text { : } 95 \% \text { confidence interval; NC: not calculated; N: number of individuals in } \\
\text { each group. }\end{array}$} \\
\hline
\end{tabular}

\section{Discussion}

Innate immune receptors are essentials in the sensing of infectious organisms, continuously monitoring the extracellular milieu as well as intracellular compartments. An example of such molecules is NOD-like receptors $(35,36)$. This family of receptors contains important components of the inflammasomes, multiprotein complexes of receptors, and sensors that mediate the innate immune response and induce inflammation (37). Several studies of genes involved in assembling the complex of inflammasomes have attempted to explain their role in the heterogeneity of diseases. For the same infection, some individuals are more susceptible to developing the disease while others remain asymptomatic (23). We showed here that the $\mathrm{C} / \mathrm{C}$ genotype $(P=0.026)$ or the allele $\mathrm{C}(P=0.002)$ in NLRP3 rs1539019 polymorphism were associated with the risk of hospitalization in SARS-CoV-2 infected individuals. In contrast, the $\mathrm{C} / \mathrm{G}$ genotype ( $P=0.016)$ or carrier-G $(P=0.028)$ in NLRP3 rs 10754558 polymorphism or carrying the A-C-G-CG haplotype $(P=0.030)$ in NLRP3 genetic variants were associated with protection of SARS-CoV-2 infected individuals against hospitalization. Our data suggest that these SNPs might modulate inflammasome activation, contributing to the risk for and protection against hospitalization, respectively.

The NLRP3 inflammasome - also known as NALP3 and cryopyrin - is currently the most studied inflammasomes component, being considered the main study model of these cytoplasmic complexes. 口The NLRP3 gene is located on the long arm of chromosome 1q44 and reacts to a diverse set of endogenous or exogenous stimuli (37). NLRP3 has been linked to the pathogenesis of several diseases, including (1) metabolic disorders, such as type 2 diabetes (38), obesity (39), autoimmune and inflammatory diseases (40-42), neurological diseases (43), and (2) diseases caused by viral pathogens, such as HIV (44), Influenza A (45), and SARS-CoV (46). SNPs in the NLRP3 gene have already been associated with a group of inflammatory disorders of genetic origin (26). Other inflammasomes and molecules related to the activation cascade (e.g., CARD8, AIM2, IFI16, CASP-1, and IL-1 $\beta$ ) have also been 
associated with a variety of infections and metabolic diseases. They may affect the function of the NLRP3 inflammasome $(47,48)$. In a recent study from our group, we verified the association of a SNP in the CARD8 gene (rs2043211) with the protection against the immune reconstitution inflammatory syndrome (IRIS) associated with HIV-TB coinfection (de Sá et al, 2021 unpublished data).

The NLRP3 rs1539019 polymorphism is an intronic variation which function still is not entirely understood. However, several studies have reported that intronic polymorphisms may be associated with susceptibility/resistance to several diseases such as rheumatoid arthritis (49), type II diabetes (50), and coronary artery disease (51). One explanation for this is that many transcription factors bind to intronic sites that may play a role in regulating gene expression. In the study by Chung et al. (2020) allele C in NLRP3 rs1539019 polymorphism was associated with the risk of renal cell carcinoma (52). Additionally, Estfanous et al. (2019) reported that rs1539019 is associated with susceptibility to hepatitis C and lower response to IFN treatment, depending on the allele and/or genotype. Moreover, Dehghan et al. (2009) reported la statistically significant association between NLRP3 rs 1539019 polymorphism and risk for cardiovascular disease $(53,54)$. As of our knowledge, our study is the first to demonstrate the association of the genotype CC and the allele C in NLRP3 rs1539019 polymorphism with the risk for hospitalization in SARS-CoV-2 infected individuals.

The NLRP3 rs10754558 polymorphism has been described by Hitomi et al. (2009) as responsible for the increasing NLRP3 mRNA stability, conferring greater expression of this gene (55). Furthermore, a Brazilian study found an association between rs $10754558 \mathrm{G}$ allele and protection against HIV-1 infection (56), while another Brazilian group showed the NLRP3 rs10754558 C/C genotype influence on the IL-1 $\beta$ cytokine serum levels (57). Several studies report the association of this polymorphism with distinct infectious diseases (58-61); however, our study is the first to show an association in SARS-CoV-2 infection. Concerning functional studies, Rodrigues et al. (2020), showed that the inflammasome is strongly active in patients with COVID-19 who require hospitalization (30). Therefore, we hypothesize that the increased expression of NLRP3, conferred by the greater stability of rs10754558 variant (55), could increase inflammasome assembly and, consequently, promote a more robust initial inflammatory response against SARS-CoV-2.

As far as we know, this is the first study demonstrating the association of inflammasome NLRP3 genetic variants with the risk and/or protection against hospitalization among SARS-CoV-2 infected individuals. Studies linking the NLRP3 inflammasome and SARS-CoV-2 infection are still scarce due to the recent emergence of this pathogen. A recent study showed that the inflammasome is robustly activated in SARS-CoV-2 infected hospitalized individuals (30). In addition, several other studies indicate that the inflammasome may be involved in the pathogenesis of the disease $(12,27-30)$. Further studies putting together genetic polymorphisms and functional analysis of the inflammasomes will be of foremost relevance to better understand the role of this cytoplasmatic protein complex in the outcomes of the SARS-CoV-2 infection.

\section{Conclusion}


The present study is the first to report the association of genetic polymorphisms in NLRP3 (rs1539019 and rs10754558) with risk and/or protection against hospitalization in SARS-CoV-2 infected individuals. We conclude that inflammasome genetic variants influence the COVID-19 clinical outcomes among patients included in our study. Functional studies are needed to better understand the role of these polymorphisms. Our work highlights the importance of the genetic variations in inflammasome genes in the clinical evolution of COVID-19.

\section{Abbreviations}

ACE2 - angiotensin-converting enzyme 2

AIM2 - interferon-inducible protein AIM2

CARD8 - caspase recruitment domain-containing protein 8

CASP-1 - caspase 1

$\mathrm{Cl}$ - confidence interval

HIV-TB - human immunodeficiency virus - tuberculosis

IFI16 - interferon gamma-inducible protein 16

IFN - interferon

IL-1 $\beta$ - interleukin 1 beta

INI - National Institute of Infectology

IOC - Oswaldo Cruz Institute

IQR - interquartile range

IRB - Institutional Review Board

NF-kB - factor nuclear kappa B

NLR - NOD-like receptors

NLRP3 - NLR family pyrin domain containing 3

PRR - pattern-recognition receptors

RLRs - retinoic acid-inducible gene--llike receptors

RT-PCR - reverse transcription polymerase chain reaction 
SNPs - single-base polymorphisms

TMPRSS2 - angiotensin I-converting enzyme-2

WHO - World Health Organization

\section{Declarations}

\section{Ethics approval and consent to participate}

This study was approved by the Ethics Committee of National Institute of Infectology Evandro Chagas (INI)/Oswaldo Cruz Foundation (FIOCRUZ), Rio de Janeiro, Brazil under the Approval number CAAE 32449420.4.1001.5262 and the Oswaldo Cruz Institute (IOC)/Oswaldo Cruz Foundation (FIOCRUZ), Rio de Janeiro, Brazil under the Approval number CAAE 68118417.6.0000.5248. Informed Consent was obtained from all study participants or their families. Anonymity was guaranteed and the data collected was kept and handled by the research team alone. All methods were performed in accordance with the relevant guidelines and regulations.

\section{Consent for publication}

Not applicable.

\section{Availability of data and materials}

The databases used and/or analyzed during the current study would be available from the corresponding author on reasonable request after anonymization.

\section{Competing interests}

The authors declare that they have no competing interests.

\section{Funding}

The study was supported by Fundação Carlos Chagas Filho de Amparo à Pesquisa do Estado do Rio de Janeiro - FAPERJ (Grant number SEl-260003/002689/2020) and INOVA FIOCRUZ/Fundação Oswaldo Cruz (Grant number 48401996705881 and 48402179262880 ). NBRS and CCG are recipients of INOVA FIOCRUZ/Fundação Oswaldo Cruz, MGM is recipient of CNPQ (314064/2018-4) and FAPERJ (E26/201.177/2021), and BG is recipient of CNPQ (305789/2019-8) and FAPERJ (E-26/202.915/2018). The funding agencies played no role in the design of the study, data collection, analysis, or interpretation, nor in writing the manuscript.

\section{Authors' contributions}

NBRDS, MNG, ASC, and LRG - Organized the samples and databank, performed the experiments; NBRDS, MNG, MRA, and MGM- Analyzed and interpreted data; MRA- Performed the statistical analyses; NBRDS, 
MNG, MRA, and MGM - Wrote the manuscript; KMG, MPDR, SWC, BG, VGV - contributed to the acquisition of data for the patients. KMG, MPDR, SWC, BG, VGV, AC, MMS, OCLB, CCG, LRG, ASC, DVA, CBGG, and FHC- revised the manuscript; NBRDS and MGM - Designed the experiments; MGM and FHC- Conceived, supervised and provided infrastructure for the entire study. All authors read and agreed with the contents and submission of this manuscript.

\section{Acknowledgements}

The authors are thankful to all patients who agreed to participate in this study and their families, the frontline health care worker of the INI/Fiocruz Hospital, and the Recover study team in Rio de Janeiro. We are in debt to Sylvia Lopes Maia Teixeira for support.

\section{References}

1. Lu H, Stratton CW, Tang YW. Outbreak of pneumonia of unknown etiology in Wuhan, China: The mystery and the miracle. J Med Virol. 2020 Apr 1;92(4):401-2.

2. Zhu N, Zhang D, Wang W, Li X, Yang B, Song J, et al. A novel coronavirus from patients with pneumonia in China, 2019. N Engl J Med. 2020;382(8):727-33.

3. World Health Organization (WHO). Laboratory testing of human suspected cases of novel coronavirus (nCoV) infection. 2020.

4. World Health Organization (WHO). Situation Report-51 SITUATION IN NUMBERS total and new cases in last 24 hours. 2020.

5. WHO. Coronavirus disease (COVID-19) Situation Report-172. 2020.

6. Worldometer. COVID-19 coronavirus pandemic. [Internet]. 2021 [cited 2021 Sep 13]. Available from: https://www.worldometers.info/coronavirus/

7. Delatorre E, Mir D, Gräf T, Bello G. Tracking the onset date of the community spread of SARS-CoV-2 in western countries. Mem Inst Oswaldo Cruz [Internet]. 2020 [cited 2020 Sep 10];115. Available from: http://www.scielo.br/scielo.php?script=sci_arttext\&pid=S0074-02762020000100415\&tlng=en

8. Ritchie H, Mathieu E, Rodés-Guirao L, Appel C, Giattino C, Ortiz-Ospina E, et al. Coronavirus Pandemic (COVID-19). Our World Data [Internet]. 2020 Mar 5 [cited 2021 Sep 12]; Available from: https://ourworldindata.org/coronavirus

9. Meintrup D, Borgmann S, Seidl K, Stecher M, Jakob CEM, Pilgram L, et al. Clinical Medicine Specific Risk Factors for Fatal Outcome in Critically III COVID-19 Patients: Results from a European Multicenter Study. J Clin Med [Internet]. 2021;10:3855. Available from: https://doi.org/10.3390/jcm10173855

10. Shi J, Zhao Y, Wang K, Shi X, Wang Y, Huang H, et al. Cleavage of GSDMD by inflammatory caspases determines pyroptotic cell death. Nature. 2015 Oct 29;526(7575):660-5.

11. Zhang H, Zeng L, Xie M, Liu J, Zhou B, Wu R, et al. TMEM173 Drives Lethal Coagulation in Sepsis. Cell Host Microbe. 2020 Apr 8;27(4):556-570.e6. 
12. Berg DF van den, Velde AA te. Severe COVID-19: NLRP3 Inflammasome Dysregulated. Front Immunol [Internet]. 2020 [cited 2021 Jan 21];11. Available from: /pmc/articles/PMC7332883/?report=abstract

13. Park A, Iwasaki A. Type I and Type III Interferons - Induction, Signaling, Evasion, and Application to Combat COVID-19. Cell Host Microbe [Internet]. 2020 Jun 10 [cited 2021 Oct 13];27(6):870. Available from: /pmc/articles/PMC7255347/

14. de Wit E, van Doremalen N, Falzarano D, Munster VJ. SARS and MERS: recent insights into emerging coronaviruses. Nat Rev Microbiol 2016148 [Internet]. 2016 Jun 27 [cited 2021 Oct 13];14(8):523-34. Available from: https://www.nature.com/articles/nrmicro.2016.81

15. Calado MB, da Silva Santana CE, Crovella S. Do inflammasome impact COVID-19 severity? VirusDisease [Internet]. 2021 Jul 26 [cited 2021 Aug 3]; Available from:

https://link.springer.com/10.1007/s13337-021-00705-3

16. Tang D et al. The hallmarks of COVID-19 disease. PLoS Pathog [Internet]. 2020 [cited 2020 Jun 4];16(5):e1008536. Available from: http://www.ncbi.nlm.nih.gov/pubmed/32442210

17. Tay MZ et al. The trinity of COVID-19: immunity, inflammation and intervention. Vol. 20, Nature Reviews Immunology. Nature Research; 2020. p. 363-74.

18. Chen G, Wu D, Guo W, Cao Y, Huang D, Wang H, et al. Clinical and immunologic features in severe and moderate Coronavirus Disease 2019. J Clin Invest. 2020;0-30.

19. Huang C, Wang Y, Li X, Ren L, Zhao J, Hu Y, et al. Clinical features of patients infected with 2019 novel coronavirus in Wuhan, China. www.thelancet.com [Internet]. 2020 [cited 2020 Apr 6];395:497. Available from: https://isaric.tghn.org/protocols/

20. Aziz M, Fatima R, Assaly R. Elevated interleukin-6 and severe COVID-19: A meta-analysis. J Med Virol [Internet]. 2020 Nov 1 [cited 2021 Oct 13];92(11):2283-5. Available from:

/pmc/articles/PMC7267383/

21. Rathinam VAK, Fitzgerald KA. Inflammasome Complexes: Emerging Mechanisms and Effector Functions. Cell [Internet]. 2016 May 5 [cited 2019 Sep 13];165(4):792-800. Available from: https://www.sciencedirect.com/science/article/pii/S0092867416303440

22. Man SM, Kanneganti T-D. Regulation of inflammasome activation. Immunol Rev [Internet]. 2015 May 1 [cited 2019 Sep 13];265(1):6-21. Available from: http://doi.wiley.com/10.1111/imr.12296

23. Figueira MB de A, de Lima DS, Boechat AL, Filho MG do N, Antunes IA, Matsuda J da S, et al. SingleNucleotide Variants in the AIM2 - Absent in Melanoma 2 Gene (rs1103577) Associated With Protection for Tuberculosis. Front Immunol. 2021 Apr 1;12.

24. Keyel PA. How is inflammation initiated? Individual influences of IL-1, IL-18 and HMGB1. Vol. 69, Cytokine. Academic Press; 2014. p. 136-45.

25. Van Reeth K. Cytokines in the pathogenesis of influenza. In: Veterinary Microbiology. Elsevier; 2000. p. 109-16.

26. Lasigliè D, Traggiai E, Federici S, Alessio M, Buoncompagni A, Accogli A, et al. Role of IL-1 beta in the development of human TH17 cells: Lesson from NLPR3 mutated patients. PLoS One. 2011;6(5). 
27. Hoel H, Heggelund L, Reikvam D, Stiksrud B, Ueland T, Michelsen A, et al. Elevated markers of gut leakage and inflammasome activation in COVID-19 patients with cardiac involvement. J Intern Med [Internet]. 2020 Sep 25 [cited 2020 Oct 12]; Available from: /pmc/articles/PMC7536991/? report=abstract

28. Amor S, Fernández Blanco L, Baker D. Innate immunity during SARS-CoV-2: evasion strategies and activation trigger hypoxia and vascular damage. Clin Exp Immunol [Internet]. 2020 Sep 26 [cited 2020 Oct 12]; Available from: /pmc/articles/PMC7537271/?report=abstract

29. Elhabyan A, Elyaacoub S, Sanad E, Abukhadra A, Elhabyan A, Dinu V. The role of host genetics in susceptibility to severe viral infections in humans and insights into host genetics of severe COVID19: A systematic review. Virus Res [Internet]. 2020;289:198163. Available from: https://doi.org/10.1016/j.virusres.2020.198163

30. Rodrigues TS, de Sá KSG, Ishimoto AY, Becerra A, Oliveira S, Almeida L, et al. Inflammasomes are activated in response to SARS-Cov-2 infection and are associated with COVID-19 severity in patients. J Exp Med. 2020;218(3).

31. Wulandari L, Hamidah B, Pakpahan C, Damayanti NS, Kurniati ND, Adiatmaja CO, et al. Initial study on TMPRSS2 p.Val160Met genetic variant in COVID-19 patients. Hum Genomics [Internet]. 2021 Dec 1 [cited 2021 Oct 13];15(1). Available from: /pmc/articles/PMC8127183/

32. Hou Y, Zhao J, Martin W, Kallianpur A, Chung MK, Jehi L, et al. New insights into genetic susceptibility of COVID-19: an ACE2 and TMPRSS2 polymorphism analysis. [cited 2020 Aug 22]; Available from: https://doi.org/10.1186/s12916-020-01673-z

33. Velavan TP, Pallerla SR, Rüter J, Augustin Y, Kremsner PG, Krishna S, et al. Host genetic factors determining COVID-19 susceptibility and severity. EBioMedicine [Internet]. 2021 Oct 1 [cited 2021 Nov 3];72. Available from: http://www.thelancet.com/article/S2352396421004229/fulltext

34. Instituto Brasileiro de Geografia e Estatística. Características étnico - raciais da população: classificação e identidades. Estudos e Análises: informação demográfica e socioeconômica. 2013. 83-99 p.

35. Medzhitov R, Janeway C. J. Advances in immunology: Innate immunity. Vol. 343, New England Journal of Medicine. 2000. p. 338-44.

36. Gong T, Liu L, Jiang W, Zhou R. DAMP-sensing receptors in sterile inflammation and inflammatory diseases. Nat Rev Immunol. 2019 Sep 26;

37. Martinon F, Mayor A, Tschopp J. The inflammasomes: guardians of the body. Annu Rev Immunol [Internet]. 2009;27:229-65. Available from: http://www.ncbi.nlm.nih.gov/pubmed/19302040

38. Yaribeygi H, Mohammadi MT, Rezaee R, Sahebkar A. Fenofibrate improves renal function by amelioration of NOX-4, IL-18, and p53 expression in an experimental model of diabetic nephropathy. J Cell Biochem [Internet]. 2018 Sep 1 [cited 2020 Apr 6];119(9):7458-69. Available from: http://doi.wiley.com/10.1002/jcb.27055

39. Kim HY, Lee HJ, Chang YJ, Pichavant M, Shore SA, Fitzgerald KA, et al. Interleukin-17-producing innate lymphoid cells and the NLRP3 inflammasome facilitate obesity-associated airway 
hyperreactivity. Nat Med. 2014;20(1):54-61.

40. Mangan MSJ, Olhava EJ, Roush WR, Seidel HM, Glick GD, Latz E. Erratum: Targeting the NLRP3 inflammasome in inflammatory diseases (Nature reviews. Drug discovery (2018) 178 (588-606)). Vol. 17, Nature reviews. Drug discovery. NLM (Medline); 2018. p. 688.

41. Alencar JB de, Zacarias JMV, Tsuneto PY, Souza VH de, Silva C de $O$ e, Visentainer JEL, et al. Influence of inflammasome NLRP3, and IL1B and IL2 gene polymorphisms in periodontitis susceptibility. PLoS One [Internet]. 2020 Jan 1 [cited 2021 Sep 13];15(1). Available from: /pmc/articles/PMC6980600/

42. Manuel Sánchez-Maldonado J, Martínez-Bueno M, Canhão H, ter Horst R, Muñoz-Peña S, Moñiz-Díez A, et al. NFKB2 polymorphisms associate with the risk of developing rheumatoid arthritis and response to TNF inhibitors: Results from the REPAIR consortium. Sci Reports 2020101 [Internet]. 2020 Mar 9 [cited 2021 Sep 13];10(1):1-13. Available from: https://www.nature.com/articles/s41598-020-61331-5

43. von Herrmann KM, Salas LA, Martinez EM, Young AL, Howard JM, Feldman MS, et al. NLRP3 expression in mesencephalic neurons and characterization of a rare NLRP3 polymorphism associated with decreased risk of Parkinson's disease. npj Park Dis 201841 [Internet]. 2018 Aug 15 [cited 2021 Sep 13];4(1):1-9. Available from: https://www.nature.com/articles/s41531-018-0061-5

44. Pontillo A, Brandão L a, Guimarães RL, Segat L, Athanasakis E, Crovella S. A 3'UTR SNP in NLRP3 gene is associated with susceptibility to HIV-1 infection. J Acquir Immune Defic Syndr. 2010;54(3):236-40.

45. Allen IC, Scull MA, Moore CB, Holl EK, McElvania-TeKippe E, Taxman DJ, et al. The NLRP3 inflammasome mediates in vivo innate immunity to influenza $A$ virus through recognition of viral RNA. Immunity [Internet]. 2009 Apr 17 [cited 2019 Nov 2];30(4):556-65. Available from: http://www.ncbi.nlm.nih.gov/pubmed/19362020

46. Wu X, Li Y, Song C-B, Chen Y-L, Fu Y-J, Jiang Y-J, et al. Increased Expression of sST2 in Early HIV Infected Patients Attenuated the IL-33 Induced T Cell Responses. Front Immunol [Internet]. 2018 Dec 4 [cited 2019 Aug 15];9:2850. Available from:

https://www.frontiersin.org/article/10.3389/fimmu.2018.02850/full

47. Leal VNC, Reis EC, Pontillo A. Inflammasome in HIV infection: Lights and shadows. Vol. 118, Molecular Immunology. Elsevier Ltd; 2020. p. 9-18.

48. Ito $S$, Hara Y, Kubota T. CARD8 is a negative regulator for NLRP3 inflammasome, but mutant NLRP3 in cryopyrin-associated periodic syndromes escapes the restriction. Arthritis Res Ther. 2014 Feb 12;16(1).

49. Pakzad B, Yousefisadr F, Karimzadeh H, Mousavi M, Noormohamadi E, Salehi R. Single nucleotide polymorphism rs5029937 in TNFAIP3 gene is correlated with risk of rheumatoid arthritis. Med J Islam Repub Iran [Internet]. 2021 Apr 30 [cited 2021 Sep 8];35:42. Available from: /pmc/articles/PMC8271223/ 
50. Lehman DM, Fu D-J, Freeman AB, Hunt KJ, Leach RJ, Johnson-Pais T, et al. A Single Nucleotide Polymorphism in MGEA5 Encoding O-GIcNAc-selective N-Acetyl- $\beta$-d Glucosaminidase Is Associated With Type 2 Diabetes in Mexican Americans. Diabetes [Internet]. 2005 Apr 1 [cited 2021 Sep 8];54(4):1214-21. Available from: https://diabetes.diabetesjournals.org/content/54/4/1214

51. S A, AN A-N, A A-S, JF B, EV B, MS A-M, et al. Intronic Polymorphisms in the CDKN2B-AS1 Gene Are Strongly Associated with the Risk of Myocardial Infarction and Coronary Artery Disease in the Saudi Population. Int J Mol Sci [Internet]. 2016 Mar 17 [cited 2021 Sep 8];17(3). Available from: https://pubmed.ncbi.nlm.nih.gov/26999117/

52. Chung C-J, Bao B-Y, Lin Y-C, Huang Y-L, Shiue H-S, Ao P-L, et al. Polymorphism of nucleotide binding domain-like receptor protein 3 (NLRP3) increases susceptibility of total urinary arsenic to renal cell carcinoma. Sci Rep [Internet]. 2020 Dec 1 [cited 2021 Aug 25];10(1). Available from: /pmc/articles/PMC7171170/

53. Estfanous SZK, Ali SA, Seif SM, Soror SHA, Abdelaziz DHA. Inflammasome genes' polymorphisms in Egyptian chronic hepatitis C patients: Influence on vulnerability to infection and response to treatment. Mediators Inflamm. 2019;2019.

54. Dehghan A, Yang Q, Peters A, Basu S, Bis JC, Rudnicka AR, et al. Association of Novel Genetic Loci with Circulating Fibrinogen Levels: A Genome-Wide Association Study in Six Population-Based Cohorts: Dehghan Genome-wide Association Study on Fibrinogen. Circ Cardiovasc Genet [Internet]. 2009 Apr [cited 2021 Aug 25];2(2):125. Available from: /pmc/articles/PMC2764985/

55. Hitomi Y, Ebisawa M, Tomikawa M, Imai T, Komata T, Hirota T, et al. Associations of functional NLRP3 polymorphisms with susceptibility to food-induced anaphylaxis and aspirin-induced asthma. J Allergy Clin Immunol. 2009;124(4).

56. Pontillo A, Oshiro TM, Girardelli M, Kamada AJ, Crovella S, Duarte AJS. Polymorphisms in Inflammasome' Genes and Susceptibility to HIV-1 Infection. BASIC Transl Sci. 2012;59(2):121-5.

57. Toro DM, Ramasawmy R, Silva Neto PV, Pereira GL, Sarmento PS, Dray HLSN, et al. Inflammasome genes polymorphisms may influence the development of hepatitis $\mathrm{C}$ in the Amazonas, Brazil. Ciccacci C, editor. PLoS One [Internet]. 2021 Jun 23 [cited 2021 Jun 30];16(6):e0253470. Available from: https://dx.plos.org/10.1371/journal.pone.0253470

58. Ravimohan S, Nfanyana K, Tamuhla N, Tiemessen CT, Weissman D, Bisson GP. Common Variation in NLRP3 Is Associated With Early Death and Elevated Inflammasome Biomarkers Among Advanced HIV/TB Co-infected Patients in Botswana. Open Forum Infect Dis. 2018;1-8.

59. Pontillo A, Brandao L, Guimaraes R, Segat L, Araujo J, Crovella S. Two SNPs in NLRP3 gene are involved in the predisposition to type-1 diabetes and celiac disease in a pediatric population from northeast Brazil. Autoimmunity. 2010 Dec;43(8):583-9.

60. Hanaei S, Sadr M, Rezaei A, Shahkarami S, Ebrahimi Daryani N, Bidoki AZ, et al. Association of NLRP3 single nucleotide polymorphisms with ulcerative colitis: A case-control study. Clin Res Hepatol Gastroenterol. 2018 Jun 1;42(3):269-75. 
61. Shen C, Wang QZ, Shen ZY, Yuan HY, Yu WJ, Chen XD, et al. Genetic association between the NLRP3 gene and acne vulgaris in a Chinese population. Clin Exp Dermatol [Internet]. 2019 Mar 1 [cited 2021 Aug 25];44(2):184-9. Available from: https://www.onlinelibrary.wiley.com/doi/full/10.1111/ced.13657

\section{Supplementary Files}

This is a list of supplementary files associated with this preprint. Click to download.

- Supplementarymaterial.docx 